\title{
The Westerbork HI survey of spiral and irregular galaxies
}

\section{HI observations of early-type disk galaxies ${ }^{\star}$}

\author{
E. Noordermeer ${ }^{1}$, J. M. van der Hulst ${ }^{1}$, R. Sancisi ${ }^{1,2}$, R. A. Swaters ${ }^{3}$, and T. S. van Albada ${ }^{1}$ \\ ${ }^{1}$ Kapteyn Astronomical Institute, University of Groningen, PO Box 800, 9700 AV Groningen, The Netherlands \\ e-mail: edo@astro.rug.nl \\ 2 INAF-Osservatorio Astronomico di Bologna, via Ranzani 1, 40127 Bologna, Italy \\ 3 Department of Astronomy, University of Maryland, College Park, MD 20742-2421, USA
}

Received 1 April 2005 / Accepted 29 June 2005

\section{ABSTRACT}

We present HI observations of 68 early-type disk galaxies from the WHISP survey. They have morphological types between S0 and Sab and absolute $B$-band magnitudes between -14 and -22 . These galaxies form the massive, high surface-brightness extreme of the disk galaxy population, few of which have been imaged in HI before. The HI properties of the galaxies in our sample span a large range; the average values of $M_{\mathrm{HI}} / L_{B}$ and $D_{\mathrm{HI}} / D_{25}$ are comparable to the ones found in later-type spirals, but the dispersions around the mean are larger. No significant differences are found between the S0/S0a and the Sa/Sab galaxies. Our early-type disk galaxies follow the same HI mass-diameter relation as later-type spiral galaxies, but their effective HI surface densities are slightly lower than those found in later-type systems. In some galaxies, distinct rings of $\mathrm{HI}$ emission coincide with regions of enhanced star formation, even though the average gas densities are far below the threshold of star formation derived by Kennicutt (1989, ApJ, 344, 685). Apparently, additional mechanisms, as yet unknown, regulate star formation at low surface densities. Many of the galaxies in our sample have lopsided gas morphologies; in most cases this can be linked to recent or ongoing interactions or merger events. Asymmetries are rare in quiescent galaxies. Kinematic lopsidedness is rare, both in interacting and isolated systems. In the appendix, we present an atlas of the HI observations: for all galaxies we show HI surface density maps, global profiles, velocity fields and radial surface density profiles.

Key words. surveys - galaxies: fundamental parameters - galaxies: ISM - galaxies: kinematics and dynamics - galaxies: spiral

\section{Introduction}

The discovery that HI rotation curves of spiral galaxies remain flat far outside the regions where the luminous mass is concentrated (Bosma 1978), has led to the now widely accepted view that galaxies are embedded in large halos of invisible matter (Faber \& Gallagher 1979; Bosma 1981; van Albada \& Sancisi 1986). The nature of this dark matter still remains a mystery, and poses one of the most persistent challenges to present day astronomy.

A systematic study of rotation curves in spiral galaxies, covering a large range of luminosities, morphological types and surface brightnesses, is crucial for a proper understanding of the distribution of dark matter in galaxies. Most HI studies in recent years have, however, focused on late-type, lowluminosity systems (de Blok et al. 1996; Swaters et al. 2002); HI observations of high-luminosity and early-type disk galaxies are rare. The only study so far aimed at a systematic investigation of $\mathrm{HI}$ rotation curves over the full range of morphological

* Appendix A is only available in electronic form at http://www.edpsciences.org and at the CDS via anonymous ftp to cdsarc.u-strasbg.fr $(130.79 .128 .5)$ or via http://cdsweb.u-strasbg.fr/cgi-bin/qcat?]/A+A/442/137 types was that by Broeils (1992). However, in his sample of 23 galaxies, only one was of morphological type earlier than $\mathrm{Sb}$ and only four had $V_{\max }>250 \mathrm{~km} \mathrm{~s}^{-1}$. S0 and Sa galaxies were also under-represented in the study by Persic et al. (1996); their Universal Rotation Curve is based on over 1000 rotation curves of which only 2 are of type Sab or earlier.

The main reason for the lack of information on the HI properties of early-type disks is the fact that they generally have a low HI flux (Roberts \& Haynes 1994). The only large-scale HI survey directed specifically at S0 and Sa galaxies was carried out by van Driel (1987), but his study was severely hampered by the low signal-to-noise ratio of his data. Jore (1997) obtained deep VLA observations for a sample of Sa galaxies, but focused mostly on asymmetries and evidence for interactions and mergers (Haynes et al. 2000).

Early-type disk galaxies form the high-mass, high-surfacebrightness end of the disk galaxy population (Roberts \& Haynes 1994). While the late-type spiral and irregular galaxies at the other end of the population are generally believed to be dominated by dark matter (Carignan \& Freeman 1988; Broeils 1992; Swaters 1999), little yet is known about the relative importance of dark and luminous matter in early-type disks. Knowledge of their dark matter content is a crucial step 
towards a proper understanding of the systematics of dark matter in galaxies.

The work presented here is part of a larger study aimed at investigating the properties of dark matter in early-type disk galaxies and its relation with the luminous components. A major part of our study consists of mapping the distribution and kinematics of neutral hydrogen in a large sample of these systems. As part of the WHISP project, we have observed 68 galaxies with morphological types between S0 and Sab. WHISP (Westerbork HI survey of spiral and irregular galaxies; Kamphuis et al. 1996; van der Hulst et al. 2001) is a survey of galaxies in the northern sky using the Westerbork Synthesis Radio Telescope (WSRT), aiming at a systematic investigation of the HI content and large-scale kinematics of disk galaxies. Observations were carried out between 1993 and 2002, and the survey now contains data for almost 400 galaxies, covering Hubble types from S0 to Im. Earlier results from WHISP were presented by Swaters et al. (2002, hereafter Paper I), Swaters \& Balcells (2002, Paper II) and García-Ruiz et al. (2002).

In this paper, we present the first results of the HI observations of the early-type disk galaxies. We discuss the basic HI properties of these galaxies and we present an atlas of HI surface density maps, velocity fields, global profiles and radial surface density distributions. The data presented here will be used in a forthcoming paper to derive rotation curves, which will then be combined with optical data to study the dark matter content and distribution in these systems.

The structure of this paper is as follows. In Sect. 2 we describe the selection of the sample and some basic properties of the selected targets. In Sect. 3, the observations and data reduction steps are discussed. In Sect. 4, we describe the HI properties of our sample galaxies, and compare them to their optical properties. Notes on individual galaxies are given in Sect. 5. Section 6 presents a summary of the main conclusions. The atlas of the HI observations is presented in the appendix.

\section{Sample selection}

All galaxies presented here were selected from the WHISP survey (Kamphuis et al. 1996; van der Hulst et al. 2001). The galaxies in WHISP were selected from the Uppsala General Catalogue of Galaxies (UGC; Nilson 1973). To ensure that the galaxies would be well resolved with the WSRT, target galaxies were selected on the basis of their position on the sky $\left(\delta>20^{\circ}\right)$, and angular size $\left(D_{25}>1^{\prime}\right)$. A lower line flux limit, defined as $f=F_{\mathrm{HI}} / W_{20}$ with $F_{\mathrm{HI}}$ the total flux in Jy km s${ }^{-1}$ and $W_{20}$ the width of the HI profile in $\mathrm{km} \mathrm{s}^{-1}$, was chosen to ensure that the channel maps have a sufficient signal-to-noise ratio to produce HI surface density maps and velocity fields. For the largest part of the survey, carried out before 1999, this limit was set at $f>100 \mathrm{mJy}$. This led to the inclusion of only 11 earlytype galaxies, compared to over 300 intermediate and late-type galaxies. In 1999, a more powerful correlator and cooled frontends on all WSRT telescopes improved the sensitivity for $21 \mathrm{~cm}$ line observations by approximately a factor of 3 . For the observations with the new setup, we lowered the flux limit to $f>20 \mathrm{mJy}$.
From the final WHISP sample, we selected all 68 galaxies with morphological type between S0 and Sab. Some basic properties of these galaxies are listed in Table 1.

Our sample is not intended to be complete in any sense, but it is still interesting to compare some general properties of our objects to the full sample of galaxies in the UGC. In Fig. 1, we show in bold lines the distribution of our sample galaxies over a number of parameters. We also show the distribution of the entire UGC catalogue (thin lines), as well as all galaxies from the UGC with morphological type between S0 and Sab (dashed lines).

The histogram for morphological type simply reflects our selection criterion for early-type disk galaxies. Compared to the entire early-type disk subsample from the UGC, we have a fair sample of Sa and Sab galaxies; S0's are however underrepresented in our sample. This is due to the well-known fact that S0 galaxies have generally an even lower HI content than Sa's (Roberts \& Haynes 1994); even with the current high sensitivity of the WSRT, only a handful of S0 galaxies can be observed in single $12 \mathrm{~h}$ runs.

Due to our diameter and HI flux limits, we select predominantly nearby galaxies, as is visible in the top-middle histogram in Fig. 1. Most of our sample galaxies have $V_{\text {hel }} \leq 5000 \mathrm{~km} \mathrm{~s}^{-1}$.

Compared to the average early-type disk galaxies in the UGC, our objects are on average slightly more gas-rich (probed by the $m_{21}-m_{\mathrm{B}}$ "color") and marginally bluer. The distribution of average B-band surface brightness of our galaxies, defined within the 25th magnitude isophote, shows a small excess on the bright side, but this is hardly significant. Similarly, we have a small excess of low-luminosity systems.

In conclusion, our sample contains a fair number of Sa and Sab galaxies, whose properties seem to resemble those of average early-type disk galaxies in the UGC very well. Their bluer color and higher gas content may indicate a higher level of star formation, but the differences for both parameters are small. The fact that the average surface brightness and luminosity distributions are almost identical to those for the entire subsample of early-type disks from the UGC, confirms our conjecture that we are not looking at a special subclass of these systems. The situation for S0's is less clear, due to the small number contained in this sample.

\section{Observations and data reduction}

All observations described here were carried out with the Westerbork Synthesis Radio Telescope (WSRT) in single $12 \mathrm{~h}$ sessions. 11 galaxies were observed before 1999, using the old front-ends and correlator. The remaining 57 were observed after the major upgrade of the WSRT, using new cooled frontends on all telescopes and a new broadband correlator. A summary of the observational parameters is given in Table 2 .

For most observations, the field of view was centered on the target galaxy. In a few cases, more than one galaxy was observed in one pointing, and some galaxies lie off-center in the field of view (notably UGC 94, 499, 508, 6621, 8699 and 12815). The angular resolution depends on the coverage of the UV-plane during the observations. Furthermore, as the WSRT is an east-west array, the resolution in declination depends on 
Table 1. WHISP early-type disk galaxy sample: basic data. (1) UGC number, (2) alternative name, (3) RA, (4) Dec, (5) morphological type, (6) absolute $B$-band magnitude, (7) heliocentric radial velocity, (8) distance, (9) inclination angle (superscripts indicate source: ${ }^{t}$ tilted ring fits to velocity fields, ${ }^{L}$ LEDA and ${ }^{e}$ estimated from our optical data) and (10) group/cluster membership (superscripts indicate source: ${ }^{1}$ Garcia (1993), ${ }^{2}$ Tully (1988), ${ }^{3}$ Tully (1980), ${ }^{4}$ Hickson et al. (1989) and ${ }^{5}$ this study). Columns (3)-(5) were taken from NED, Col. (6) from LEDA and Cols. (7) and (8) from this study (cf. Table 3).

\begin{tabular}{|c|c|c|c|c|c|c|c|c|c|c|c|c|c|}
\hline \multirow{2}{*}{$\begin{array}{c}\overline{\text { UGC }} \\
(1)\end{array}$} & \multirow{2}{*}{$\begin{array}{l}\text { Alternative } \\
\text { name } \\
(2)\end{array}$} & \multicolumn{3}{|c|}{ RA (2000) } & \multicolumn{3}{|c|}{ Dec (2000) } & \multirow{2}{*}{$\begin{array}{c}\text { Type } \\
(5)\end{array}$} & \multirow{2}{*}{$\begin{array}{c}M_{\mathrm{B}} \\
\mathrm{mag} \\
(6)\end{array}$} & \multirow{2}{*}{$\begin{array}{c}V_{\text {hel }} \\
\mathrm{km} \mathrm{s}^{-1} \\
(7)\end{array}$} & \multirow{2}{*}{$\begin{array}{c}D \\
\mathrm{Mpc} \\
(8)\end{array}$} & \multirow{2}{*}{$\begin{array}{c}i \\
\circ \\
(9)\end{array}$} & \multirow{2}{*}{$\begin{array}{c}\text { Group/cluster } \\
\text { membership } \\
\text { (10) }\end{array}$} \\
\hline & & $\mathrm{h}$ & $\begin{array}{l}\mathrm{m} \\
(3)\end{array}$ & $\mathrm{s}$ & & (4) & " & & & & & & \\
\hline 89 & NGC 23 & 0 & 9 & 53.4 & 25 & 55 & 26 & $\mathrm{SB}(\mathrm{s}) \mathrm{a}$ & -21.48 & 4555 & 62.1 & $50^{t}$ & LGG $2^{1}$ \\
\hline 94 & NGC 26 & 0 & 10 & 25.9 & 25 & 49 & 55 & $\mathrm{SA}(\mathrm{rs}) \mathrm{ab}$ & -20.21 & 4589 & 62.6 & $42^{t}$ & LGG $2^{1}$ \\
\hline 232 & - & 0 & 24 & 38.7 & 33 & 15 & 22 & $\mathrm{SB}(\mathrm{r}) \mathrm{a}$ & -19.96 & 4839 & 66.3 & $48^{\mathrm{L}}$ & - \\
\hline 499 & NGC 262 & 0 & 48 & 47.1 & 31 & 57 & 25 & $\mathrm{SA}(\mathrm{s}) 0 / \mathrm{a}:$ & -20.09 & 4534 & 62.0 & $46^{\mathrm{L}}$ & LGG $14^{1}$ \\
\hline 508 & NGC 266 & 0 & 49 & 47.8 & 32 & 16 & 40 & $\mathrm{SB}(\mathrm{rs}) \mathrm{ab}$ & -21.97 & 4647 & 63.6 & $25^{\mathrm{t}}$ & LGG $14^{1}$ \\
\hline 624 & NGC 338 & 1 & 0 & 36.4 & 30 & 40 & 8 & $\mathrm{Sab}$ & -21.42 & 4772 & 65.1 & $59^{t}$ & LGG $14^{1}$ \\
\hline 798 & IC 1654 & 1 & 15 & 11.9 & 30 & 11 & 41 & (R)SB(r)a & -19.67 & 4896 & 66.7 & $40^{t}$ & LGG $18^{1}$ \\
\hline 1310 & NGC 694 & 1 & 50 & 58.5 & 21 & 59 & 51 & S0? pec & -19.23 & 2958 & 40.2 & $47^{\mathrm{e}}$ & LGG $34^{1}$ \\
\hline 1541 & NGC 797 & 2 & 3 & 27.9 & 38 & 7 & 1 & $\mathrm{SAB}(\mathrm{s}) \mathrm{a}$ & -21.10 & 5649 & 77.0 & $41^{\mathrm{t}}$ & pair with NGC $801^{5}$ \\
\hline 2045 & NGC 972 & 2 & 34 & 13.4 & 29 & 18 & 41 & $\mathrm{Sab}$ & -20.39 & 1527 & 21.4 & $61^{\mathrm{e}}$ & $52-0^{2}$ (Cetus) \\
\hline 2141 & NGC 1012 & 2 & 39 & 14.9 & 30 & 9 & 6 & SO/a? & -18.02 & 987 & 14.3 & $90^{\mathrm{e}}$ & $17-3^{2}$ (Triang. Spur) \\
\hline 2154 & NGC 1023 & 2 & 40 & 24.0 & 39 & 3 & 48 & $\mathrm{SB}(\mathrm{rs}) 0-$ & -20.23 & 695 & 10.9 & $64^{\mathrm{L}}$ & N 1023 group $^{3}$ \\
\hline 2183 & NGC 1056 & 2 & 42 & 48.3 & 28 & 34 & 27 & Sa: & -19.60 & 1540 & 21.5 & $90^{\mathrm{e}}$ & - \\
\hline 2487 & NGC 1167 & 3 & 1 & 42.4 & 35 & 12 & 21 & SA0- & -21.73 & 4950 & 67.4 & $36^{\mathrm{t}}$ & LGG $80^{1}$ \\
\hline 2916 & - & 4 & 2 & 33.8 & 71 & 42 & 21 & Sab & -20.34 & 4518 & 63.5 & $46^{t}$ & LGG $115^{1}$ \\
\hline 2941 & IC 357 & 4 & 3 & 44.0 & 22 & 9 & 33 & $\mathrm{SB}(\mathrm{s}) \mathrm{ab}$ & -21.38 & 6261 & 83.9 & $45^{\mathrm{e}}$ & group with UGC $2942 / 43^{5}$ \\
\hline 2953 & IC 356 & 4 & 7 & 46.9 & 69 & 48 & 45 & SA(s)ab pec & -21.49 & 894 & 15.1 & $50^{\mathrm{t}}$ & $12+15^{2}$ (U. Major) \\
\hline 3205 & - & 4 & 56 & 14.8 & 30 & 3 & 8 & $\mathrm{Sab}$ & -21.47 & 3587 & 48.7 & $67^{\mathrm{t}}$ & - \\
\hline 3354 & - & 5 & 47 & 18.2 & 56 & 6 & 44 & Sab: & -20.00 & 3084 & 43.6 & $90^{\mathrm{e}}$ & - \\
\hline 3382 & - & 5 & 59 & 47.7 & 62 & 9 & 29 & $\mathrm{SB}(\mathrm{rs}) \mathrm{a}$ & -20.62 & 4501 & 62.8 & $16^{\mathrm{t}}$ & - \\
\hline 3407 & - & 6 & 9 & 8.1 & 42 & 5 & 7 & $\mathrm{Sa}$ & -20.05 & 3606 & 49.8 & $45^{\mathrm{e}}$ & - \\
\hline 3426 & - & 6 & 15 & 36.3 & 71 & 2 & 15 & S0: & -20.89 & 4005 & 56.6 & $37^{\mathrm{e}}$ & LGG $135^{1}$ \\
\hline 3546 & NGC 2273 & 6 & 50 & 8.7 & 60 & 50 & 45 & $\mathrm{SB}(\mathrm{r}) \mathrm{a}$ & -20.16 & 1838 & 27.3 & $52^{\mathrm{t}}$ & $24-1^{2}(\operatorname{Lynx})$ \\
\hline 3580 & - & 6 & 55 & 30.8 & 69 & 33 & 47 & SA(s)a pec: & -18.19 & 1200 & 19.2 & $63^{\mathrm{t}}$ & $12-0^{2}$ (U. Major) \\
\hline 3642 & - & 7 & 4 & 20.3 & 64 & 1 & 13 & SA0 & -21.00 & 4498 & 62.9 & $45^{\mathrm{t}}$ & LGG $140^{1}$ \\
\hline 3965 & IC 2204 & 7 & 41 & 18.1 & 34 & 13 & 56 & (R)SB(r)ab & -18.64 & 4588 & 62.5 & $10^{\mathrm{e}}$ & - \\
\hline 3993 & - & 7 & 55 & 44.0 & 84 & 55 & 35 & S0? & -19.61 & 4366 & 61.9 & $20^{t}$ & pair with UGC $3992^{5}$ \\
\hline 4458 & NGC 2599 & 8 & 32 & 11.3 & 22 & 33 & 38 & SAa & -21.12 & 4757 & 64.2 & $27^{\mathrm{t}}$ & pair with PGC $23972^{5}$ \\
\hline 4605 & NGC 2654 & 8 & 49 & 11.9 & 60 & 13 & 16 & SBab: sp & -20.08 & 1350 & 20.9 & $90^{\mathrm{e}}$ & $13-6^{2}$ (U. Major S. Spur) \\
\hline 4637 & NGC 2655 & 8 & 55 & 37.7 & 78 & 13 & 23 & $\mathrm{SAB}(\mathrm{s}) 0 / \mathrm{a}$ & -20.97 & 1415 & 22.4 & $30^{\mathrm{e}}$ & $12-10^{2}$ (U. Major) \\
\hline 4666 & NGC 2685 & 8 & 55 & 34.7 & 58 & 44 & 4 & (R)SB0+ pec & -19.21 & 876 & 14.6 & $61^{\mathrm{L}}$ & $13-4^{2}$ (U. Major S. Spur) \\
\hline 4862 & NGC 2782 & 9 & 14 & 5.1 & 40 & 6 & 49 & $\mathrm{SAB}(\mathrm{rs}) \mathrm{a}$ & -20.99 & 2540 & 35.9 & $20^{\mathrm{e}}$ & $21-0^{2}$ (Leo) \\
\hline 5060 & NGC 2893 & 9 & 30 & 17.0 & 29 & 32 & 24 & (R)SB0/a & -18.14 & 1699 & 24.1 & $24^{e}$ & $21-0^{2}($ Leo $)$ \\
\hline 5253 & NGC 2985 & 9 & 50 & 22.2 & 72 & 16 & 43 & $\left(\mathrm{R}^{\prime}\right) \mathrm{SA}(\mathrm{rs}) \mathrm{ab}$ & -20.72 & 1325 & 21.1 & $38^{\mathrm{t}}$ & $12-7^{2}$ (U. Major) \\
\hline 5351 & NGC 3067 & 9 & 58 & 21.0 & 32 & 22 & 12 & $\mathrm{SAB}(\mathrm{s}) \mathrm{ab} ?$ & -19.35 & 1486 & 21.5 & $70^{\mathrm{L}}$ & $21-12^{2}($ Leo $)$ \\
\hline 5559 & NGC 3190 & 10 & 18 & 5.6 & 21 & 49 & 55 & $\mathrm{SA}(\mathrm{s}) \mathrm{a}$ pec $\mathrm{sp}$ & -19.88 & 1308 & 18.6 & $67^{\mathrm{L}}$ & $\mathrm{HCG} 44^{4}$ \\
\hline 5906 & NGC 3380 & 10 & 48 & 12.2 & 28 & 36 & 07 & $\left(\mathrm{R}^{\prime}\right) \mathrm{SBa}$ ? & -18.69 & 1601 & 23.1 & $29^{\mathrm{e}}$ & LGG $227^{1}$ \\
\hline 5960 & NGC 3413 & 10 & 51 & 20.7 & 32 & 45 & 59 & So & -17.39 & 643 & 10.5 & $77^{\mathrm{t}}$ & - \\
\hline 6001 & NGC 3442 & 10 & 53 & 8.1 & 33 & 54 & 37 & $\mathrm{Sa} ?$ & -17.77 & 1730 & 25.1 & $47^{\mathrm{L}}$ & $21-9^{2}($ Leo $)$ \\
\hline 6118 & NGC 3504 & 11 & 3 & 11.2 & 27 & 58 & 21 & (R)SAB(s)ab & -20.28 & 1536 & 22.2 & $24^{\mathrm{e}}$ & $21-7^{2}($ Leo $)$ \\
\hline 6283 & NGC 3600 & 11 & 15 & 52.0 & 41 & 35 & 29 & $\mathrm{Sa} ?$ & -17.17 & 713 & 12.0 & $90^{\mathrm{e}}$ & $15-9^{2}$ (Leo Spur) \\
\hline 6621 & NGC 3786 & 11 & 39 & 42.5 & 31 & 54 & 33 & $\left(\mathrm{R}^{\prime}\right) \mathrm{SAB}(\mathrm{r})$ a pec & -19.74 & 2742 & 38.7 & $58^{\mathrm{L}}$ & $13-0^{2}$ (U. Major S. Spur) \\
\hline 6623 & NGC 3788 & 11 & 39 & 44.6 & 31 & 55 & 52 & $\mathrm{SAB}(\mathrm{rs}) \mathrm{ab}$ pec & -20.24 & 2670 & 37.7 & $74^{\mathrm{L}}$ & $13-0^{2}$ (U. Major S. Spur) \\
\hline 6742 & NGC 3870 & 11 & 45 & 56.6 & 50 & 12 & 00 & S0? & -17.28 & 752 & 13.0 & $36^{\mathrm{L}}$ & $12-1^{2}$ (U. Major) \\
\hline 6786 & NGC 3900 & 11 & 49 & 9.4 & 27 & 1 & 19 & $\mathrm{SA}(\mathrm{r}) 0+$ & -19.94 & 1799 & 25.9 & $65^{\mathrm{t}}$ & $13-9^{2}$ (U. Major S. Spur) \\
\hline 6787 & NGC 3898 & 11 & 49 & 15.4 & 56 & 5 & 4 & $\mathrm{SA}(\mathrm{s}) \mathrm{ab}$ & -20.25 & 1171 & 18.9 & $67^{\mathrm{t}}$ & $12-3^{2}$ (U. Major) \\
\hline 7166 & NGC 4151 & 12 & 10 & 32.6 & 39 & 24 & 21 & (R')SAB(rs)ab: & -20.84 & 998 & 15.9 & $20^{t}$ & $12-6^{2}$ (U. Major) \\
\hline 7256 & NGC 4203 & 12 & 15 & 5.0 & 33 & 11 & 50 & SAB0-: & -19.47 & 1091 & 16.9 & $40^{t}$ & $14-1^{2}$ (Coma-Sculptor) \\
\hline 7489 & NGC 4369 & 12 & 24 & 36.2 & 39 & 22 & 59 & (R)SA(rs)a & -18.87 & 1027 & 16.4 & $17^{\mathrm{L}}$ & $12-6^{2}$ (U. Major) \\
\hline 7506 & NGC 4384 & 12 & 25 & 12.0 & 54 & 30 & 22 & $\mathrm{Sa}$ & -19.70 & 2532 & 37.0 & $41^{\mathrm{L}}$ & $42+11^{2}$ (Canes Venatici) \\
\hline 7704 & NGC 4509 & 12 & 33 & 6.8 & 32 & 5 & 30 & Sab pec? & -16.17 & 937 & 14.8 & $60^{\mathrm{e}}$ & LGG $279^{1}$ \\
\hline 7989 & NGC 4725 & 12 & 50 & 26.6 & 25 & 30 & 3 & $\mathrm{SAB}(\mathrm{r}) \mathrm{ab}$ pec & -21.69 & 1208 & 18.2 & $51^{\mathrm{t}}$ & $14-2^{2}$ (Coma-Sculptor) \\
\hline 8271 & NGC 5014 & 13 & 11 & 31.2 & 36 & 16 & 55 & $\mathrm{Sa} ? \mathrm{sp}$ & -18.44 & 1128 & 17.7 & $60^{\mathrm{e}}$ & $43-1^{2}$ (Canes V. Spur) \\
\hline
\end{tabular}


Table 1. continued.

\begin{tabular}{|c|c|c|c|c|c|c|c|c|c|c|c|c|c|}
\hline \multirow{2}{*}{$\begin{array}{l}\text { UGC } \\
(1) \\
\end{array}$} & \multirow{2}{*}{$\begin{array}{l}\text { alternative } \\
\text { name } \\
(2)\end{array}$} & \multicolumn{3}{|c|}{ RA (2000) } & \multicolumn{3}{|c|}{ Dec (2000) } & \multirow{2}{*}{$\begin{array}{l}\text { Type } \\
(5)\end{array}$} & \multirow{2}{*}{$\begin{array}{c}M_{\mathrm{B}} \\
\mathrm{mag} \\
(6) \\
\end{array}$} & \multirow{2}{*}{$\begin{array}{c}V_{\text {hel }} \\
\mathrm{km} \mathrm{s}^{-1} \\
(7)\end{array}$} & \multirow{2}{*}{$\begin{array}{c}D \\
\mathrm{Mpc} \\
(8) \\
\end{array}$} & \multirow{2}{*}{$\begin{array}{c}i \\
\circ \\
(9)\end{array}$} & \multirow{2}{*}{$\begin{array}{c}\text { Group/cluster } \\
\text { membership } \\
(10)\end{array}$} \\
\hline & & $\mathrm{h}$ & $\begin{array}{r}\mathrm{m} \\
\text { (3) }\end{array}$ & $\mathrm{s}$ & $\circ$ & (4) & " & & & & & & \\
\hline 8699 & NGC 5289 & 13 & 45 & 8.7 & 41 & 30 & 12 & (R)SABab: & -19.70 & 2521 & 36.7 & $72^{t}$ & LGG $361^{1}$ \\
\hline 8805 & NGC 5347 & 13 & 53 & 17.8 & 33 & 29 & 27 & $\left(\mathrm{R}^{\prime}\right) \mathrm{SB}(\mathrm{rs}) \mathrm{ab}$ & -19.69 & 2384 & 34.5 & $30^{\mathrm{e}}$ & $42-0^{2}$ (Canes Venatici) \\
\hline 8863 & NGC 5377 & 13 & 56 & 16.7 & 47 & 14 & 8 & $(\mathrm{R}) \mathrm{SB}(\mathrm{s}) \mathrm{a}$ & -20.32 & 1791 & 27.2 & $51^{t}$ & $42-0^{2}$ (Canes Venatici) \\
\hline 9133 & NGC 5533 & 14 & 16 & 7.7 & 35 & 20 & 38 & $\mathrm{SA}(\mathrm{rs}) \mathrm{ab}$ & -21.47 & 3861 & 54.3 & $53^{t}$ & LGG $380^{1}$ \\
\hline 9644 & - & 14 & 59 & 34.3 & 27 & 6 & 58 & $\mathrm{SB}(\mathrm{r}) \mathrm{a}$ & -19.25 & 6665 & 91.5 & $19^{\mathrm{e}}$ & - \\
\hline 10448 & NGC 6186 & 16 & 34 & 25.5 & 21 & 32 & 27 & $\left(\mathrm{R}^{\prime}\right) \mathrm{SB}(\mathrm{s}) \mathrm{a}$ & -21.65 & 11351 & 153.8 & $30^{\mathrm{e}}$ & - \\
\hline 11269 & NGC 6667 & 18 & 30 & 39.8 & 67 & 59 & 13 & SABab? pec & -20.11 & 2581 & 38.3 & $56^{\mathrm{L}}$ & $70-0^{2}(-)$ \\
\hline 11670 & NGC 7013 & 21 & 3 & 33.6 & 29 & 53 & 51 & $\mathrm{SA}(\mathrm{r}) 0 / \mathrm{a}$ & -19.53 & 775 & 12.7 & $68^{t}$ & $65+5^{2}$ (Pegasus Spur) \\
\hline 11852 & - & 21 & 55 & 59.3 & 27 & 53 & 54 & SBa? & -20.25 & 5846 & 80.0 & $50^{t}$ & - \\
\hline 11914 & NGC 7217 & 22 & 7 & 52.4 & 31 & 21 & 33 & (R)SA(r)ab & -20.45 & 949 & 14.9 & $31^{t}$ & $65+1^{2}$ (Pegasus Spur) \\
\hline 11951 & NGC 7231 & 22 & 12 & 30.1 & 45 & 19 & 42 & $\mathrm{SBa}$ & -19.14 & 1086 & 17.4 & $70^{\mathrm{L}}$ & - \\
\hline 12043 & NGC 7286 & 22 & 27 & 50.5 & 29 & 5 & 45 & $\mathrm{~S} 0 / \mathrm{a}$ & -17.15 & 1007 & 15.4 & $67^{t}$ & - \\
\hline 12276 & NGC 7440 & 22 & 58 & 32.5 & 35 & 48 & 9 & $\mathrm{SB}(\mathrm{r}) \mathrm{a}$ & -20.59 & 5662 & 77.7 & $37^{\mathrm{L}}$ & - \\
\hline 12713 & - & 23 & 38 & 14.4 & 30 & 42 & 29 & $\mathrm{~S} 0 / \mathrm{a}$ & -14.35 & 295 & 5.7 & $72^{\mathrm{t}}$ & $65+4^{2}$ (Pegasus Spur) \\
\hline 12815 & NGC 7771 & 23 & 51 & 24.9 & 20 & 6 & 43 & $\mathrm{SB}(\mathrm{s}) \mathrm{a}$ & -21.60 & 4307 & 58.5 & $61^{\mathrm{L}}$ & LGG $483^{1}$ \\
\hline
\end{tabular}
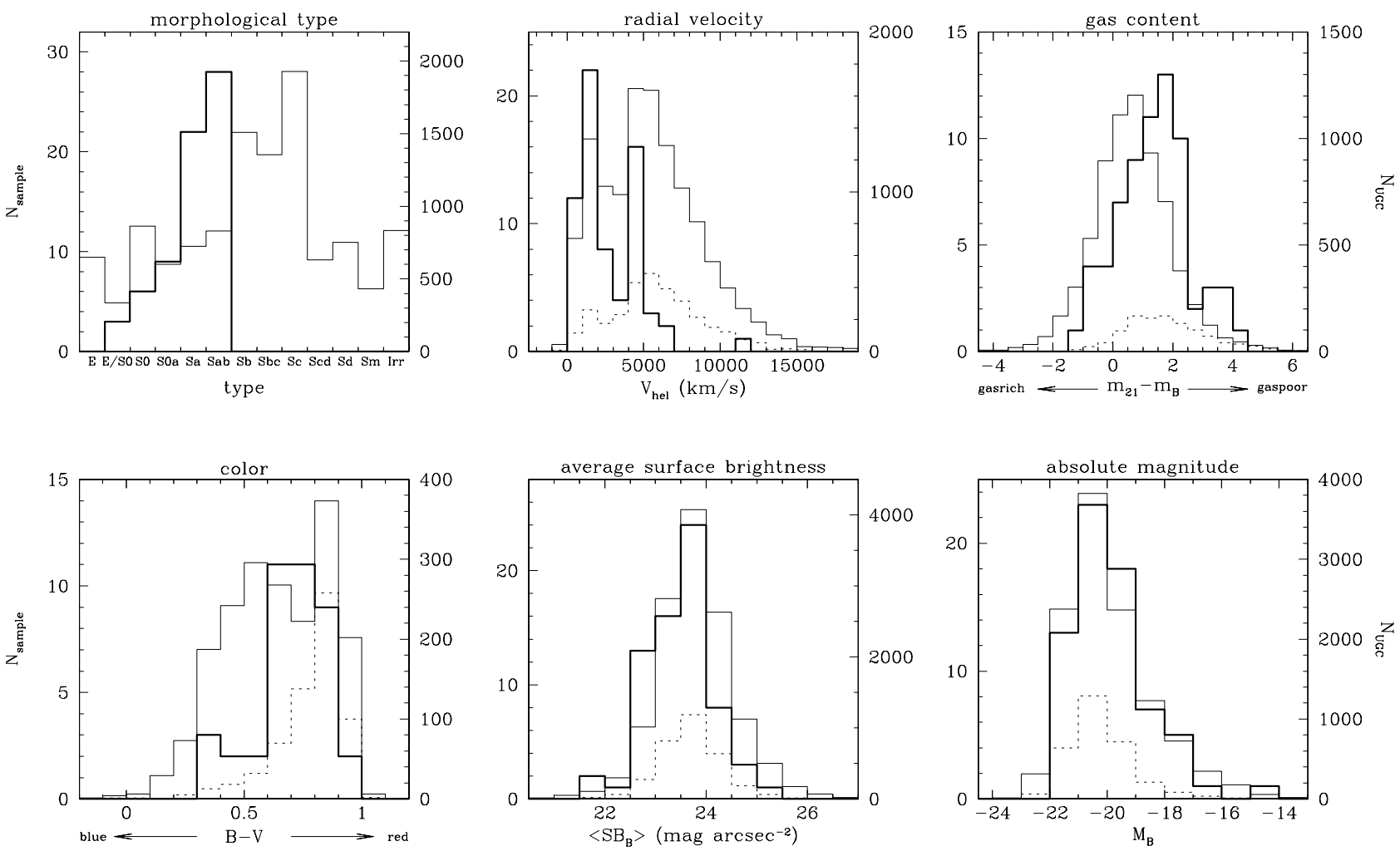

Fig. 1. Characteristics of our sample galaxies (bold lines), compared with the entire UGC (thin lines) and with all galaxies from the UGC with type between S0 and Sab (dashed lines). The scales on the left of each panel give the distribution of the galaxies in the sample presented here, the scales on the right apply to the galaxies from the UGC. All data are taken from LEDA ${ }^{1}$, except the heliocentric velocities for our sample galaxies, which are taken from this study. Note that some parameters (e.g. color, gas content) are not available for all galaxies in LEDA; the total number of galaxies included is different for each histogram.

the position on the sky and scales as $1 / \sin \delta$. The resulting beam size for each galaxy is given in Table 2.

Observations were usually done with 128 channels; the total bandwidth was chosen on the basis of the HI line width $W_{20}$, such that we had optimal wavelength resolution while still having a reasonable baseline to construct continuum maps. The resulting velocity resolution, before Hanning smoothing, is thus either $\sim 2.5,5,10$ or $20 \mathrm{~km} \mathrm{~s}^{-1}$, with the exact value depending on the central wavelength of the band (see Table 2).

The data reduction consisted of several stages; each will be described below.

1 LEDA: Lyon Extragalactic Database,

http://leda.univ-lyon1.fr/ 
Table 2. Observational parameters: (1) UGC number, (2) observation date, (3) spatial resolution of the unsmoothed data, (4) velocity resolution before Hanning smoothing, (5) rms noise level in the unsmoothed channel maps, (6) 1 sigma column density in the HI maps shown in the atlas, (7) previous $21 \mathrm{~cm}$ synthesis observations (references are explained at the bottom of the table) and (8) remarks.

\begin{tabular}{|c|c|c|c|c|c|c|c|c|}
\hline \multirow[t]{3}{*}{ UGC } & \multirow[t]{2}{*}{ Observation date } & \multicolumn{3}{|c|}{ Resolution } & \multirow{4}{*}{$\begin{array}{c}\sigma_{\mathrm{ch}} \\
\mathrm{mJy} / \mathrm{beam} \\
(5)\end{array}$} & \multirow{4}{*}{$\begin{array}{c}\sigma_{\text {map }} \\
10^{19} \text { atoms cm }{ }^{-2} \\
(6)\end{array}$} & \multirow{4}{*}{$\begin{array}{c}\begin{array}{c}\text { Previous } \\
\text { observations }\end{array} \\
\text { (7) }\end{array}$} & \multirow{4}{*}{$\begin{array}{r}\text { Remarks } \\
\text { (8) }\end{array}$} \\
\hline & & RA & Dec & $v$ & & & & \\
\hline & & $" \prime$ & $"$ & $\mathrm{~km} \mathrm{~s}^{-1}$ & & & & \\
\hline (1) & (2) & \multicolumn{2}{|c|}{ (3) } & (4) & & & & \\
\hline 89 & Sep. 2000 & 16.1 & 37.9 & 10.03 & 0.64 & 3.25 & $\mathrm{O} 93$ & \\
\hline 94 & Sep. 2000 & 16.1 & 37.9 & 10.03 & 0.64 & 2.86 & O93 & 1 pointing \\
\hline 232 & July 2000 & 15.7 & 29.9 & 10.04 & 0.78 & 4.97 & - & \\
\hline 499 & Aug. 2000 & 11.1 & 19.4 & 10.04 & 0.69 & 2.56 & H82, S87 & \\
\hline 508 & Aug. 2000 & 11.1 & 19.4 & 10.04 & 0.69 & 2.82 & - & \}$^{1}$ pointing \\
\hline 624 & July 2000 & 10.7 & 24.2 & 10.04 & 0.61 & 11.3 & - & \\
\hline 798 & Aug. 2000 & 12.6 & 23.2 & 10.04 & 0.73 & 3.13 & - & \\
\hline 1310 & June 2000 & 12.0 & 31.8 & 9.98 & 0.90 & 3.66 & VM88 & \\
\hline 1541 & Aug. 2000 & 16.1 & 27.0 & 10.07 & 0.79 & 7.06 & - & \\
\hline 2045 & Aug. 2000 & 15.9 & 34.0 & 9.94 & 0.67 & 2.75 & $\mathrm{H} 03$ & \\
\hline 2141 & Jan. $1994^{\dagger}$ & 8.3 & 19.4 & 4.98 & 3.51 & 71.8 & - & \\
\hline 2154 & July $1997^{\dagger}$ & 10.1 & 18.0 & 19.80 & 1.20 & 2.88 & A79, S84 & \\
\hline 2183 & Apr. 2000 & 10.6 & 26.0 & 9.94 & 0.79 & 6.52 & - & \\
\hline 2487 & Dec. 2000 & 11.8 & 22.2 & 10.06 & 0.87 & 1.29 & - & \\
\hline 2916 & Apr. 2000 & 10.8 & 13.2 & 10.03 & 0.59 & 16.8 & - & \\
\hline 2941 & July 2000 & 10.7 & 32.3 & 5.06 & 1.31 & 6.47 & - & \\
\hline 2953 & Sep. 2001 & 10.7 & 14.4 & 9.91 & 0.60 & 1.39 & - & \\
\hline 3205 & Dec. 2000 & 10.6 & 24.5 & 10.01 & 0.61 & 15.5 & - & \\
\hline 3354 & Nov. 2000 & 16.9 & 21.1 & 9.98 & 0.57 & 5.93 & - & \\
\hline 3382 & Dec. 2000 & 12.2 & 14.1 & 5.04 & 0.75 & 1.20 & - & \\
\hline 3407 & Dec. 2000 & 12.7 & 17.8 & 10.01 & 0.71 & 2.38 & - & \\
\hline 3426 & Jan. 2002 & 12.3 & 13.0 & 5.03 & 0.54 & 1.20 & - & \\
\hline 3546 & Dec. 2000 & 12.2 & 14.9 & 9.95 & 0.63 & 1.13 & VW83, VD91 & \\
\hline 3580 & Jan. $1994^{\dagger}$ & 9.1 & 10.2 & 4.98 & 2.50 & 5.45 & B94 & \\
\hline 3642 & May 2000 & 12.6 & 13.4 & 10.03 & 0.75 & 3.69 & - & \\
\hline 3965 & Apr. 2000 & 11.7 & 21.4 & 2.51 & 1.84 & 1.95 & - & \\
\hline 3993 & July 2000 & 12.2 & 12.3 & 5.03 & 0.82 & 1.32 & - & \\
\hline 4458 & July 2002 & 15.5 & 43.6 & 10.04 & 0.74 & 3.14 & - & \\
\hline 4605 & Apr. 2000 & 12.8 & 13.7 & 9.94 & 0.69 & 18.4 & W88 & \\
\hline 4637 & July 2002 & 12.6 & 12.4 & 9.94 & 0.68 & 0.74 & - & \\
\hline 4666 & Aug. 2000 & 11.8 & 15.6 & 9.91 & 0.69 & 2.95 & S80 & \\
\hline 4862 & July 2000 & 15.5 & 24.8 & 5.00 & 0.99 & 6.07 & S94 & \\
\hline 5060 & July 2000 & 15.6 & 33.2 & 4.99 & 0.88 & 2.04 & - & \\
\hline 5253 & Aug. $1993^{\dagger}$ & 11.6 & 12.4 & 19.92 & 1.82 & 3.64 & O93 & \\
\hline 5351 & Aug. 2000 & 14.0 & 20.8 & 9.94 & 0.75 & 4.11 & C89, C92 & \\
\hline 5559 & June $1996^{\dagger}$ & 9.8 & 26.3 & 19.92 & 1.98 & 31.1 & W91 & \\
\hline 5906 & Aug. 2000 & 34.6 & 15.8 & 4.99 & 1.05 & 2.04 & - & \\
\hline 5960 & Apr. 2000 & 21.7 & 11.6 & 4.97 & 1.40 & 6.45 & - & \\
\hline 6001 & Aug. 2000 & 21.4 & 11.7 & 4.99 & 0.97 & 5.07 & - & \\
\hline 6118 & July 2000 & 35.2 & 15.7 & 9.94 & 0.78 & 4.35 & VM83b & \\
\hline 6283 & Apr. $1996^{\dagger}$ & 18.7 & 12.5 & 4.97 & 3.11 & 28.9 & GR02 & \\
\hline 6621 & July 2002 & 31.2 & 15.9 & 9.97 & 0.59 & 3.70 & O93 & \\
\hline 6623 & July 2002 & 31.2 & 15.9 & 9.97 & 0.59 & 3.70 & O93 & 1 pointing \\
\hline 6742 & Apr. 2000 & 14.1 & 12.8 & 4.97 & 0.81 & 1.84 & - & \\
\hline 6786 & Aug. 2000 & 15.5 & 36.0 & 9.95 & 0.74 & 1.48 & VD89, H00 & \\
\hline 6787 & Apr. 2000 & 12.6 & 13.8 & 9.92 & 0.65 & 2.14 & VD94 & \\
\hline 7166 & June $1997^{\dagger}$ & 20.8 & 12.9 & 4.98 & 2.98 & 1.73 & B77, P92, M99 & \\
\hline
\end{tabular}

$\dagger$ Observed before the upgrade of the WSRT.

\subsection{Standard WHISP pipeline}

The initial reduction steps were carried out following the standard WHISP data reduction pipeline. The raw UV data were inspected and calibrated using the NEWSTAR software package. Bad data-points were flagged interactively. The UV data were then Fourier transformed to the image plane, and antenna patterns were calculated. For each set of observations, data 
Table 2. continued.

\begin{tabular}{|c|c|c|c|c|c|c|c|c|}
\hline \multirow[t]{3}{*}{$\overline{\mathrm{UGC}}$} & \multirow[t]{4}{*}{ Observation date } & \multicolumn{3}{|c|}{ Resolution } & \multirow{4}{*}{$\begin{array}{c}\sigma_{\mathrm{ch}} \\
\mathrm{mJy} / \mathrm{beam} \\
(5)\end{array}$} & \multirow{4}{*}{$\begin{array}{c}\sigma_{\text {map }} \\
10^{19} \text { atoms cm } \mathrm{cm}^{-2} \\
(6)\end{array}$} & \multirow{4}{*}{$\begin{array}{c}\begin{array}{c}\text { Previous } \\
\text { observations }\end{array} \\
\text { (7) }\end{array}$} & \multirow{4}{*}{$\begin{array}{c}\text { Remarks } \\
\text { (8) }\end{array}$} \\
\hline & & RA & Dec & $v$ & & & & \\
\hline & & " & & $\mathrm{km} \mathrm{s}^{-1}$ & & & & \\
\hline$(1)$ & & \multicolumn{2}{|c|}{ (3) } & (4) & & & & \\
\hline 7256 & July 2000 & 30.7 & 15.3 & 4.98 & 1.76 & 2.29 & VD88 & \\
\hline 7489 & Aug. $1997^{\dagger}$ & 18.2 & 11.4 & 4.98 & 4.30 & 5.18 & - & \\
\hline 7506 & Aug. 2000 & 13.0 & 10.3 & 5.00 & 0.93 & 13.1 & - & \\
\hline 7704 & Aug. 2000 & 21.3 & 10.0 & 4.98 & 0.93 & 4.69 & - & \\
\hline 7989 & Oct. $1994^{\dagger}$ & 8.0 & 13.2 & 19.80 & 1.13 & 9.73 & W84 & \\
\hline 8271 & July 2000 & 10.2 & 19.9 & 4.98 & 0.91 & 0.98 & - & \\
\hline 8699 & Aug. 2000 & 16.9 & 10.7 & 9.97 & 0.68 & 12.4 & VM83a & \\
\hline 8805 & July $1997^{\dagger}$ & 19.5 & 10.6 & 5.00 & 4.41 & 2.49 & - & \\
\hline 8863 & Aug. 2000 & 15.8 & 22.4 & 9.95 & 0.66 & 2.11 & - & \\
\hline 9133 & July 2000 & 15.8 & 28.8 & 10.02 & 0.70 & 2.44 & B92, B94 & \\
\hline 9644 & July 2000 & 10.5 & 25.6 & 5.08 & 1.08 & 9.41 & - & \\
\hline 10448 & July 2000 & 10.6 & 32.1 & 5.15 & 1.10 & 5.26 & - & \\
\hline 11269 & July 2000 & 12.2 & 14.4 & 9.97 & 0.59 & 1.78 & - & \\
\hline 11670 & May 2000 & 11.3 & 25.0 & 9.91 & 0.73 & 2.37 & K84 & \\
\hline 11852 & July 2000 & 16.1 & 35.7 & 10.08 & 0.78 & 4.12 & - & \\
\hline 11914 & Sep. 2000 & 10.5 & 23.5 & 9.92 & 0.68 & 2.25 & VM95 & \\
\hline 11951 & Jan. $1994^{\dagger}$ & 10.1 & 14.1 & 4.98 & 3.04 & 54.5 & - & \\
\hline 12043 & Apr. 2000 & 9.9 & 25.9 & 4.98 & 1.22 & 9.50 & - & \\
\hline 12276 & July 2002 & 10.4 & 19.3 & 5.05 & 0.84 & 9.45 & - & \\
\hline 12713 & June 2000 & 11.6 & 22.0 & 4.97 & 0.95 & 1.78 & VD91b & \\
\hline 12815 & Aug. 2000 & 10.4 & 33.4 & 10.03 & 0.70 & 9.62 & - & \\
\hline
\end{tabular}

Observed before the upgrade of the WSRT.

Explanation of the references in col. (7):

A79 Allsopp (1979); B77 Bosma et al. (1977); B92 Broeils (1992); B94 Broeils \& van Woerden (1994); C89 Carilli et al. (1989); C92 Carilli \& van Gorkom (1992); GR02 García-Ruiz et al. (2002); H00 Haynes et al. (2000); H03 Hameed \& Young (2003); H82 Heckman et al. (1982); K84 Knapp et al. (1984); M99 Mundell et al. (1999); O93 Oosterloo \& Shostak (1993); P92 Pedlar et al. (1992); S80 Shane (1980); S84 Sancisi et al. (1984); S87 Simkin et al. (1987); S94 Smith (1994); VD88 van Driel et al. (1988); VD89 van Driel et al. (1989); VD91 van Driel \& Buta (1991); VD91b van Driel \& van Woerden (1991); VD94 van Driel \& van Woerden (1994); VM83a van Moorsel (1983a); VM83b van Moorsel (1983b); VM88 van Moorsel (1988); VM95 Verdes-Montenegro et al. (1995); VW83 van Woerden et al. (1983); W84 Wevers et al. (1984); W88 Warmels (1988a); W91 Williams et al. (1991).

cubes were created at 3 different resolutions and corresponding noise levels. One is at full resolution using all available UV data-points; the resulting beam size is given in Table 2. The other two were produced by down-weighting progressively more long baselines from the UV data. While doing so, the spatial resolution was decreased to respectively $\approx 30^{\prime \prime}$ and $\approx 60^{\prime \prime}$, but the signal-to-noise level for extended emission was strongly enhanced.

All further data reduction was done with GIPSY (Groningen Image Processing System; Vogelaar \& Terlouw 2001, http://www.astro.rug.nl/ gipsy/). For all data cubes, the continuum was subtracted by fitting, at each line of sight, a first order polynomial to the channels without HI emission. The cube at $60^{\prime \prime}$ resolution was then Hanning smoothed in velocity and masks were created by hand to identify the regions containing HI emission. The data cubes were then CLEANed (Schwarz 1978), using the masks to define the search areas. CLEANing was iterated down to 0.5 times the rms in each channel map. The rms noise in the cleaned, full resolution channel maps, $\sigma_{\text {ch }}$, is given in Table 2 .

In more than $50 \%$ of the cases, the data cubes at full resolution are of sufficient quality to derive useful HI maps and velocity fields. However, in cases where the signal is very weak, the signal-to-noise ratio in the full resolution data may be too low to extract the emission line profiles. In those cases we used the smoothed data in the subsequent analysis. Additionally, the smoothed data cubes are sometimes used to study the distribution and kinematics of the gas in the faint outer regions. In the figures in the atlas we indicate which data cube was used for each galaxy.

\subsection{Defining masks}

Before proceeding to the next steps, we defined first the regions that contain emission; parts of the data cubes that contain only noise were masked out, such that as little noise as possible entered the global profiles and surface density maps.

Creating the masks is not straightforward and requires special care. Applying a simple sigma-clipping criterion on the channel maps is not sufficient, as we may miss extended lowlevel emission. Therefore, we first smoothed the channel maps: the channel maps at full, 30 and $60^{\prime \prime}$ resolution were smoothed to 30,60 and $120^{\prime \prime}$ respectively. Subsequently the data cubes were Hanning smoothed in velocity. The signal-to-noise ratio for extended emission in the resulting smoothed data cubes is much higher and low-level emission is now easily detected. 
The Hanning-smoothed data cubes at 30,60 and 120" were then used to create masks for the original channel maps at full, 30 and $60^{\prime \prime}$ resolution respectively; the masks were created in 2 steps. First we selected all pixels in the smoothed channel maps that have intensity $>2 \sigma$. In this way, we did not only select real emission from the galaxy, but also many noise peaks in the data. In the second step we selected by hand the emission which we deemed to be real. Emission was defined to be real if it is spatially extended (i.e. $\gtrsim 2$ beam areas) and present in more than 2 adjacent channels $o r$ when it is very bright $(\gtrsim 4 \sigma)$. Small regions that are only slightly brighter than $2 \sigma$ are defined to be noise and were rejected. This second step is by definition subjective. Some peaks we judged to be noise may actually be real and vice versa. However, since we are working on extensively smoothed data, the real emission is more easily distinguished from noise, and the effect is small.

The resulting clipped channel maps were then used as masks for the original data cubes.

\subsection{Global profiles and total Hı masses}

Global profiles were derived from the data cubes at $60^{\prime \prime}$ resolution. In these data cubes, we are most sensitive to extended $\mathrm{HI}$ emission, and the flux enclosed by the masks is maximal. The global profiles were constructed by adding up all flux in each masked channel map and correcting for primary beam attenuation. Care was taken not to include flux from companion galaxies.

The error in each point of the profile is related to the size of the masked region in the channel map. Due to the non-uniform sampling of the UV-plane by the WSRT, the noise in the channel maps is not spatially independent and the error does not simply increase as the square root of the mask area. Instead, we established an empirical relation between mask size and error in the flux and used the result to estimate the errors on our global profiles.

The global profiles are shown in the top right panels in the atlas (Appendix A).

Line widths were determined at the $20 \%$ and $50 \%$ levels. If a profile was double peaked, the peaks on both sides were used separately to determine the $20 \%$ and $50 \%$ levels, otherwise the overall peak flux was used. The profile widths $W_{20}$ and $W_{50}$ were defined as the difference between the velocities at the appropriate level on each side of the profile. We followed Verheijen \& Sancisi (2001) to correct the observed line widths for instrumental broadening, assuming an internal velocity dispersion of the gas of $10 \mathrm{~km} \mathrm{~s}^{-1}$ :

$$
\begin{aligned}
& W_{20}^{c}=W_{20}-35.8\left[\sqrt{1+\left(\frac{R}{23.5}\right)^{2}}-1\right] \\
& W_{50}^{c}=W_{50}-23.5\left[\sqrt{1+\left(\frac{R}{23.5}\right)^{2}}-1\right],
\end{aligned}
$$

with $R$ the instrumental velocity resolution in $\mathrm{km} \mathrm{s}^{-1}$.

The systemic velocities $V_{\text {sys }}$ were defined as the average of the velocities at the profile edges at the $20 \%$ and $50 \%$ level. Distances $D$ were derived using a Hubble constant of
$75 \mathrm{~km} \mathrm{~s}^{-1} \mathrm{Mpc}^{-1}$, correcting the systemic velocities for Virgocentric inflow using the values given by LEDA. The profile widths, systemic velocities and distances are given in Table 3 .

The total HI mass was derived from the total flux, i.e. the integral of the global profile just derived, and is given by:

$M_{\mathrm{HI}}=236 \cdot D^{2} \cdot \int F \mathrm{~d} v$,

where $M_{\mathrm{HI}}$ is in solar masses, $D$ is the distance in Mpc, $F$ is the primary beam corrected flux in mJy, and the integral is over the total bandwidth. The derived total fluxes and $\mathrm{HI}$ masses are given in Table 3 as well.

\subsection{Integrated Hı maps}

HI maps were created by integrating the masked data cubes along the velocity direction and correcting the result for primary beam attenuation. The noise in these maps is not constant: the galaxies presented here are generally characterized by very large velocity gradients in the center and slowly varying velocities in the outer parts, so that we are summing over a variable number of channel maps at different positions. For each map, we estimated an average noise level, $\sigma_{\text {map }}$, following the prescriptions in Verheijen \& Sancisi (2001). Noise fields were constructed based on the number of channel maps contributing to the integrated $\mathrm{HI}$ maps at each position and the rms noise in the individual channel maps. Using these fields, we selected all points in our HI maps with signal-to-noise ratio between 2.75 and 3.25. The average value of these points was then divided by 3 to obtain the $\sigma_{\text {map }}$ value. It is important to note that this is an average noise level; low-level emission which is only present in a few adjacent channels may be significant, even if it is weaker than the $2 \sigma_{\mathrm{map}}$-level of the outer contour in the figures.

The HI column density maps are shown in the left hand panels in the atlas. In Table 2, we give the corresponding values of $\sigma_{\text {map }}$ in atoms $\mathrm{cm}^{-2}$.

\subsection{Velocity fields}

The two methods most commonly used to derive velocity fields from HI data cubes are to calculate the intensity weighted mean (IWM) velocity of the line profiles or to fit Gaussians to them. For the data presented here, neither of these methods proved to be adequate in recovering the true radial velocity of the gas. Particularly in the central regions of the early-type galaxies studied here, large velocity gradients are present and the line profiles are seriously skewed by beam-smearing. In those cases, both the IWM velocity and the central velocity of the fitted Gaussians can be offset significantly from the true gas velocity at the projected radius (e.g. Paper I). Here, we derive velocity fields by fitting a Gauss-Hermite polynomial that includes an h3-term for skewness to the line profiles (van der Marel \& Franx 1993). The resulting velocities from this method lie usually closer to the peak in the profile than simple IWM or Gauss fits, especially in the central regions.

We created velocity fields in two steps. In the first step, we fitted the Gauss-Hermite function to the profiles in the masked data cubes. However, since the masked data cubes contain no 
Table 3. HI properties: (1) UGC number, (2) systemic velocity, (3) distance, (4) line width of global profile at the 20\% level, (5) idem at the $50 \%$ level, (6) total HI flux, (7) total HI mass, (8) HI radius in ", (9) idem in kpc, (10) average face-on HI surface density within HI radius, (11) idem within optical radius, (12) amount of asymmetry in global profile, (13) morphological asymmetry, (14) kinematical asymmetry, and (15) evidence for current interaction/merging/accretion.

\begin{tabular}{|c|c|c|c|c|c|c|c|c|c|c|c|c|c|c|}
\hline UGC & $\begin{array}{c}V_{\text {sys }} \\
\mathrm{km} \mathrm{s}^{-1} \\
(2)\end{array}$ & $\begin{array}{c}D \\
\mathrm{Mpc} \\
(3)\end{array}$ & $\begin{array}{c}W_{20}^{c} \\
\mathrm{~km} \mathrm{~s}^{-1} \\
(4)\end{array}$ & $\begin{array}{c}W_{50}^{c} \\
\mathrm{~km} \mathrm{~s}^{-1} \\
(5)\end{array}$ & $\underset{\mathrm{Jy}}{\int \mathrm{km} \mathrm{s}^{-1}} \underset{(6)}{ }$ & $\begin{array}{c}M_{\mathrm{HI}} \\
10^{9} M_{\odot} \\
(7)\end{array}$ & $\begin{array}{c}R_{\mathrm{HI}} \\
\prime \prime \\
(8)\end{array}$ & $\begin{array}{l}R_{\mathrm{HI}} \\
\mathrm{kpc} \\
(9)\end{array}$ & $\begin{array}{c}\left\langle\Sigma_{\mathrm{HI}}\right\rangle_{R_{\mathrm{HI}}} \\
M_{\odot} \\
(10)\end{array}$ & $\begin{array}{c}\left\langle\Sigma_{\mathrm{HI}}\right\rangle_{R_{25}} \\
M_{\odot} \\
(11)\end{array}$ & $\begin{array}{l}\text { prof. } \\
\text { (12) }\end{array}$ & $\begin{array}{l}\text { Asym } \\
\text { morph. } \\
\text { (13) }\end{array}$ & $\begin{array}{l}\text { netries } \\
\text { kin. } \\
(14)\end{array}$ & $\begin{array}{c}\text { interac. } \\
(15)\end{array}$ \\
\hline 89 & 4555 & 62.1 & 440 & 386 & 9.44 & 8.63 & 77 & 24 & 3.5 & 5.1 & $*$ & $*$ & & + \\
\hline 94 & 4589 & 62.6 & 319 & 300 & 12.19 & 11.28 & 85 & 26 & 4.0 & 7.2 & $*$ & * & & + \\
\hline 232 & 4839 & 66.3 & 276 & 236 & 7.66 & 7.95 & 82 & 27 & 2.1 & 3.3 & & $*$ & & \\
\hline 499 & 4534 & 62.0 & 91 & 65 & 23.9 & 21.45 & 104 & 31 & 1.6 & 1.8 & & $* *$ & n.a. & + \\
\hline 508 & 4647 & 63.6 & 478 & 453 & 7.71 & 7.40 & 94 & 29 & 2.0 & 2.0 & & $*$ & & + \\
\hline 624 & 4772 & 65.1 & 560 & 518 & 14.39 & 14.42 & 92 & 29 & 4.3 & 7.7 & & $* *$ & $* * *$ & + \\
\hline 798 & 4896 & 66.7 & 220 & 205 & 3.88 & 4.08 & 72 & 23 & 1.8 & 2.4 & & & & \\
\hline 1310 & 2958 & 40.2 & 186 & 117 & 6.16 & 2.33 & 50 & 9.7 & 4.8 & 14.3 & $* *$ & n.a. & n.a. & + \\
\hline 1541 & 5649 & 77.0 & 446 & 421 & 7.94 & 11.14 & 75 & 28 & 2.8 & 3.9 & $* * *$ & $*$ & $*$ & \\
\hline 2045 & 1527 & 21.4 & 334 & 289 & 18.89 & 2.09 & 94 & 9.9 & 4.2 & 3.6 & & & & \\
\hline 2141 & 987 & 14.3 & 232 & 201 & 47.94 & 2.30 & 155 & 10 & 4.5 & 11.3 & & $*$ & & \\
\hline 2154 & 695 & 10.9 & 529 & 497 & 80.13 & 2.26 & $-^{\#}$ & $-\#$ & - & 0.7 & n.a. & n.a. & n.a. & + \\
\hline 2183 & 1540 & 21.5 & 292 & 268 & 32.99 & 3.63 & 164 & 17 & 3.0 & 6.8 & $*$ & $*$ & $*$ & \\
\hline 2487 & 4950 & 67.4 & 466 & 437 & 15.93 & 17.09 & 120 & 39 & 1.6 & 1.8 & & & & \\
\hline 2916 & 4518 & 63.5 & 357 & 337 & 23.08 & 21.94 & 108 & 34 & 4.3 & 7.3 & $*$ & & $* *$ & + \\
\hline 2941 & 6261 & 83.9 & 160 & 139 & 9.84 & 16.32 & 75 & 30 & 3.4 & 5.4 & & $*$ & $*$ & + \\
\hline 2953 & 894 & 15.1 & 484 & 464 & 119.73 & 6.43 & 300 & 22 & 1.9 & 2.2 & & & & \\
\hline 3205 & 3587 & 48.7 & 437 & 419 & 16.38 & 9.18 & 118 & 28 & 3.4 & 4.7 & & & & \\
\hline 3354 & 3084 & 43.6 & 423 & 398 & 17.71 & 7.95 & 77 & 16 & 2.0 & 2.3 & $*$ & & & \\
\hline 3382 & 4501 & 62.8 & 204 & 193 & 5.68 & 5.27 & 78 & 24 & 2.0 & 2.8 & & & & \\
\hline 3407 & 3606 & 49.8 & 316 & 298 & 3.13 & 1.82 & 50 & 12 & 2.6 & 3.1 & $*$ & & & + \\
\hline 3426 & 4005 & 56.6 & 161 & 114 & 3.27 & 2.53 & $-\#$ & $-\#$ & -\# & 0.2 & * & n.a. & n.a. & + \\
\hline 3546 & 1838 & 27.3 & 367 & 347 & 13.74 & 2.42 & 132 & 18 & 2.0 & 2.2 & & & & \\
\hline 3580 & 1200 & 19.2 & 241 & 224 & 43.24 & 3.76 & 199 & 18 & 3.0 & 5.4 & $*$ & & * & \\
\hline 3642 & 4498 & 62.9 & 469 & 441 & 38.13 & 35.62 & 171 & 52 & 3.1 & 4.7 & $*$ & & & \\
\hline 3965 & 4588 & 62.5 & 93 & 74 & 15.67 & 14.47 & 106 & 33 & 3.2 & 5.3 & & & & \\
\hline 3993 & 4366 & 61.9 & 209 & 192 & 7.73 & 6.99 & 82 & 24 & 1.6 & 1.7 & & $*$ & & \\
\hline 4458 & 4757 & 64.2 & 284 & 245 & 12.24 & 11.82 & 110 & 34 & 2.0 & 3.1 & & $* *$ & & + \\
\hline 4605 & 1350 & 20.9 & 432 & 392 & 58.03 & 5.99 & 277 & 28 & 1.8 & 3.0 & & & & \\
\hline 4637 & 1415 & 22.4 & 399 & 243 & 22.34 & 2.62 & ${ }^{\#}$ & $-\#$ & -\# & 0.9 & $* *$ & $* * *$ & n.a. & + \\
\hline 4666 & 876 & 14.6 & 303 & 284 & 29.07 & 1.47 & 184 & 12 & 2.2 & 2.5 & $*$ & $*$ & & \\
\hline 4862 & 2540 & 35.9 & 192 & 156 & 16.42 & 5.07 & 90 & 16 & 3.1 & 2.2 & $* * *$ & $* * *$ & n.a. & + \\
\hline 5060 & 1699 & 24.1 & 185 & 86 & 5.13 & 0.70 & 60 & 7.0 & 2.3 & 3.7 & $* *$ & $* * *$ & & \\
\hline 5253 & 1325 & 21.1 & 320 & 296 & 132.4 & 13.92 & 330 & 34 & 2.1 & 3.8 & $*$ & $* * *$ & & + \\
\hline 5351 & 1486 & 21.5 & 276 & 187 & 9.23 & 0.99 & 61 & 6.3 & 3.4 & 3.1 & $* *$ & $* *$ & $* *$ & + \\
\hline 5559 & 1308 & 18.6 & 473 & 449 & 7.32 & 0.60 & 88 & 7.7 & 2.6 & 1.5 & & n.a. & n.a. & + \\
\hline 5906 & 1601 & 23.1 & 123 & 110 & 2.53 & 0.31 & 47 & 5.3 & 2.0 & 1.9 & & $*$ & & \\
\hline 5960 & 643 & 10.5 & 180 & 154 & 28.42 & 0.75 & 109 & 5.7 & 6.4 & 15.9 & $* *$ & $*$ & $* *$ & \\
\hline 6001 & 1730 & 25.1 & 153 & 121 & 3.58 & 0.33 & 44 & 5.3 & 4.2 & 9.6 & $*$ & n.a. & n.a. & \\
\hline 6118 & 1536 & 22.2 & 219 & 194 & 7.20 & 0.84 & 89 & 9.5 & 2.7 & 3.0 & $*$ & & & \\
\hline 6283 & 713 & 12.0 & 216 & 200 & 57.38 & 2.00 & 204 & 12 & 3.1 & 5.5 & & $*$ & & \\
\hline 6621 & 2742 & 38.7 & 420 & 375 & $3.69^{\dagger}$ & $1.30^{\dagger}$ & 57 & 10 & 4.1 & 4.1 & & $* * *$ & n.a. & + \\
\hline 6623 & 2670 & 37.7 & 520 & 438 & $11.51^{\dagger}$ & $3.95^{\dagger}$ & 79 & 15 & 3.5 & 4.4 & $* * *$ & $* * *$ & n.a. & + \\
\hline 6742 & 752 & 13.0 & 127 & 84 & 4.08 & 0.17 & 51 & 3.2 & 4.3 & 8.0 & $*$ & $* *$ & & \\
\hline 6786 & 1799 & 25.9 & 445 & 423 & 24.91 & 3.93 & 143 & 18 & 2.4 & 3.4 & & & & \\
\hline 6787 & 1171 & 18.9 & 484 & 466 & 46.82 & 3.96 & 256 & 23 & 1.7 & 2.5 & $*$ & $*$ & & + \\
\hline 7166 & 998 & 15.9 & 140 & 121 & 67.58 & 4.05 & 304 & 24 & 2.1 & 2.2 & $* *$ & $*$ & $*$ & \\
\hline 7256 & 1091 & 16.9 & 271 & 239 & 48.11 & 3.21 & 226 & 19 & 1.3 & 1.8 & $*$ & $* *$ & n.a. & + \\
\hline 7489 & 1027 & 16.4 & 86 & 68 & 4.07 & 0.26 & 55 & 4.4 & 3.4 & 2.7 & & n.a. & n.a. & \\
\hline 7506 & 2532 & 37.0 & 171 & 116 & 3.66 & 1.17 & 37 & 6.7 & 4.1 & 3.7 & $*$ & n.a. & n.a. & \\
\hline 7704 & 937 & 14.8 & 93 & 61 & 6.49 & 0.34 & 58 & 4.3 & 3.7 & 9.4 & & $*$ & & \\
\hline 7989 & 1208 & 18.2 & 417 & 390 & 125.78 & 9.78 & 380 & 34 & 2.4 & 2.7 & $* * *$ & $* *$ & $*$ & + \\
\hline 8271 & 1128 & 17.7 & 166 & 76 & 10.37 & 0.78 & 89 & 7.7 & 2.1 & 3.7 & $*$ & $* * *$ & n.a. & + \\
\hline
\end{tabular}

\# Average surface density $<1 \quad M_{\odot} \mathrm{pc}^{-2}$ everywhere.

$\dagger$ Total flux and HI mass measured from $30^{\prime \prime}$ data. 
Table 3. continued.

\begin{tabular}{|c|c|c|c|c|c|c|c|c|c|c|c|c|c|c|}
\hline $\begin{array}{l}\text { UGC } \\
\text { (1) }\end{array}$ & $\begin{array}{c}V_{\text {sys }} \\
\mathrm{km} \mathrm{s}^{-1} \\
(2)\end{array}$ & $\begin{array}{c}D \\
\mathrm{Mpc} \\
(3)\end{array}$ & $\begin{array}{c}W_{20}^{c} \\
\mathrm{~km} \mathrm{~s}^{-1} \\
(4)\end{array}$ & $\begin{array}{c}W_{50}^{c} \\
\mathrm{~km} \mathrm{~s}^{-1} \\
(5)\end{array}$ & $\underset{\text { Jy } \mathrm{km} \mathrm{s}_{(6)}^{-1}}{F \mathrm{~d} v}$ & $\begin{array}{c}M_{\mathrm{HI}} \\
10^{9} M_{\odot} \\
\quad(7)\end{array}$ & $\begin{array}{c}R_{\mathrm{HI}} \\
\prime \prime \\
(8)\end{array}$ & $\begin{array}{l}R_{\mathrm{HI}} \\
\mathrm{kpc} \\
(9)\end{array}$ & $\begin{array}{c}\left\langle\Sigma_{\mathrm{HI}}\right\rangle_{R_{\mathrm{HI}}} \\
M_{\odot} \mathrm{pc}^{-2} \\
(10)\end{array}$ & $\begin{array}{c}\left\langle\Sigma_{\mathrm{HI}}\right\rangle_{R_{25}} \\
M_{\odot} \mathrm{pc}^{-2} \\
(11)\end{array}$ & $\begin{array}{l}\text { prof. } \\
\text { (12) }\end{array}$ & $\begin{array}{l}\text { Asym } \\
\text { morph. } \\
\text { (13) }\end{array}$ & $\begin{array}{l}\text { eetries } \\
\text { kin. } \\
\text { (14) }\end{array}$ & $\begin{array}{c}\text { interac. } \\
(15)\end{array}$ \\
\hline 8699 & 2521 & 36.7 & 387 & 368 & 10.22 & 3.26 & 100 & 17 & 2.9 & 4.6 & & & & \\
\hline 8805 & 2384 & 34.5 & 129 & 82 & 15.45 & 4.34 & 137 & 23 & 1.8 & 3.0 & & & & \\
\hline 8863 & 1791 & 27.2 & 391 & 367 & 14.44 & 2.52 & 148 & 19 & 1.5 & 1.6 & * & & * & \\
\hline 9133 & 3861 & 54.3 & 451 & 416 & 43.25 & 30.23 & 218 & 57 & 1.9 & 4.0 & $* *$ & $* *$ & & \\
\hline 9644 & 6665 & 91.5 & 131 & 119 & 3.63 & 7.16 & 53 & 24 & 3.2 & 4.4 & & & & \\
\hline 10448 & 11351 & 153.8 & 99 & 85 & 1.76 & 9.82 & 40 & 29 & 2.6 & 2.0 & & & & \\
\hline 11269 & 2581 & 38.3 & 415 & 344 & 31.32 & 10.86 & 161 & 30 & 3.0 & 6.1 & * & $* * *$ & $* *$ & + \\
\hline 11670 & 775 & 12.7 & 342 & 325 & 28.05 & 1.08 & 158 & 9.9 & 2.5 & 2.5 & $* *$ & & * & \\
\hline 11852 & 5846 & 80.0 & 329 & 305 & 19.55 & 29.6 & 137 & 53 & 2.5 & 4.2 & ** & & $* *$ & \\
\hline 11914 & 949 & 14.9 & 322 & 309 & 13.16 & 0.69 & 113 & 8.1 & 3.0 & 2.8 & & & & \\
\hline 11951 & 1086 & 17.4 & 222 & 201 & 25.93 & 1.85 & 127 & 10.7 & 4.4 & 9.6 & & & & + \\
\hline 12043 & 1007 & 15.4 & 190 & 176 & 20.96 & 1.18 & 143 & 10.8 & 2.4 & 6.1 & & & & \\
\hline 12276 & 5662 & 77.7 & 135 & 108 & 4.09 & 5.83 & 67 & 26 & 2.2 & 2.9 & $*$ & * & & + \\
\hline 12713 & 295 & 5.7 & 147 & 101 & 10.79 & 0.08 & 84 & 2.3 & 2.0 & 4.3 & * & * & $*$ & \\
\hline 12815 & 4307 & 58.5 & 656 & 457 & $11.74^{\$}$ & $9.36^{\$}$ & 103 & 30 & 1.9 & 2.6 & n.a. & n.a. & n.a. & + \\
\hline
\end{tabular}

$\$$ Includes emission from UGC 12813.

noise information outside the emission lines, it is difficult to determine the significance and reliability of the fitted profiles. To overcome these difficulties, we used the fitted parameters from the first step as initial estimates for a second fit to the Hanning-smoothed, unmasked line profiles. For these latter fits we could develop strict criteria to determine whether or not a profile represents true emission: only those fits were accepted that have 1) a line strength greater than 2.5 times the rms noise $\sigma_{\mathrm{h}}$ in the Hanning-smoothed channel maps and 2) a velocity within the range defined by the global profile. These two constraints were usually sufficient to exclude erroneous results. As an extra sanity check, we excluded fits with 3) a profile width smaller than 4 or larger than $200 \mathrm{~km} \mathrm{~s}^{-1}$. This criterion was, however, rarely met.

It is important to note here that the sigma-clipping criterion in 1) above was performed using the rms in the Hanningsmoothed channel maps directly. This rms is not the same as the $\sigma_{\text {map }}$ level in the HI surface density maps, which was determined empirically (see previous section). Thus, it is possible that points which fall outside the $2 \sigma_{\text {map }}$ contour in the HI density map, have a fitted line strength greater than $2.5 \sigma_{\mathrm{h}}$ and vice versa.

The final velocity fields are shown in the top middle panels in the atlas. In the bottom middle panels, we show the fitted velocities over-plotted on position-velocity slices along the major axes through the data cubes. From these figures, it is clear that the inclusion of an h3-term for skewness in the fitting procedure worked well in recovering the true velocity of the gas for most galaxies (e.g. UGC 89, 6786). In some cases, however, in particular in edge-on or poorly resolved galaxies, projection effects lead to such complicated line-profiles that even skewed Gaussians fail to recover the projected rotational velocity of the gas. In our sample, the velocity fields of UGC 1310, 2045, 5906, 5960, 6001, 6742, 7704, 8271 and 12713 suffer seriously from this effect and can therefore not be used to derive rotation curves; for UGC 2183, 3354, 4605 and 11670 , only the inner regions of the velocity fields are affected.

\subsection{Radial profiles, Hı diameters and surface densities}

The integrated HI maps created in Sect. 3.4 were used to derive radial profiles of the HI surface density. The intensities were azimuthally averaged in concentric elliptical annuli. Pixels without measured signal in the total HI map were counted as zero. The azimuthal averaging was also done separately for the approaching and receding half of the galaxy to obtain a crude estimate of the level of asymmetries present in the HI map.

The orientation of the annuli was determined with different methods, depending on the galaxy. For a number of galaxies, tilted ring fits to the velocity field were available. The details of these fits will be described in a forthcoming paper dealing with the rotation curves of our galaxies. If no tilted ring analysis was available (for example if the galaxy is too poorly resolved, or if the HI gas does not show signs of circular rotation), we used the ellipticity and position angle from LEDA. However, in some cases LEDA does not give a position angle or is clearly inconsistent with our data. Especially for near face-on galaxies, LEDA's position angles - if present - can be far off from the angles we infer from the velocity field. In those cases, we did not use LEDA's position angle, but rather determined it by eye from our HI maps and velocity fields.

The measured intensities were corrected to face-on surface densities, and converted to units of $M_{\odot} \mathrm{pc}^{-2}$. The resulting radial profiles are shown in the bottom right panels in the atlas.

For edge-on galaxies, this method does not work, as emission from different radii is superposed onto the same position on the sky. For these cases, we used the iterative Lucy deconvolution method developed by Warmels (1988b). The HI surface density maps were integrated parallel to the minor axis to get HI strip integrals. These were then converted to surface density profiles using the iterative deconvolution scheme from Lucy (1974), under the assumption of axisymmetry for the gas distribution.

From the radial profiles, HI radii were determined. The HI radius $R_{\mathrm{HI}}$ is defined as the radius where the face-on corrected 


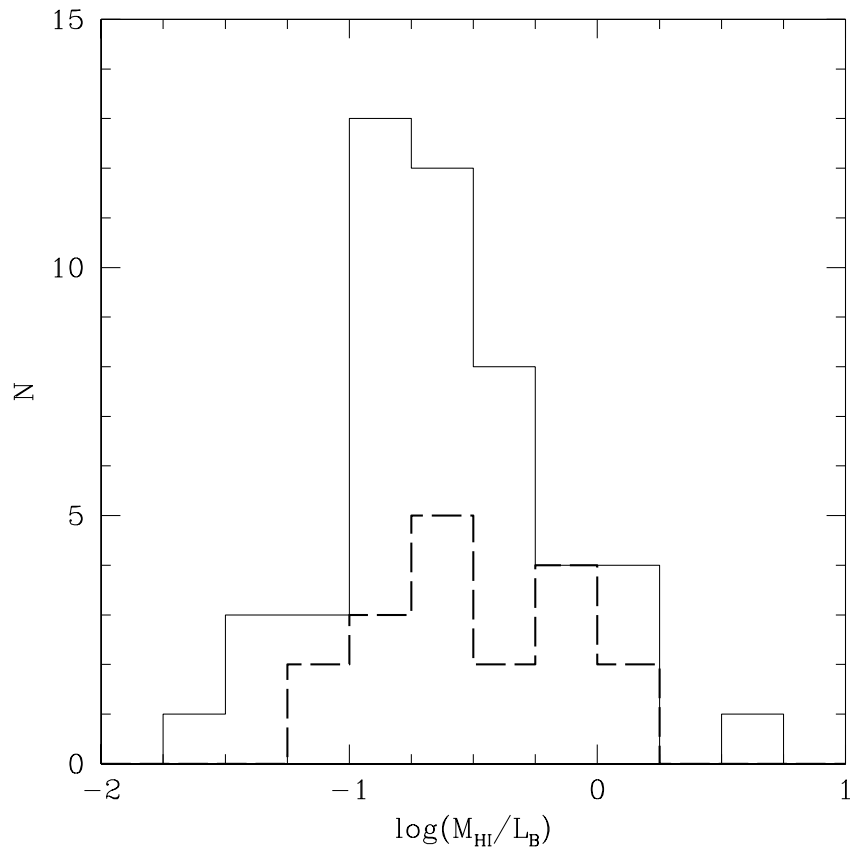

Fig. 2. The distribution of $M_{\mathrm{HI}} / L_{B}$. The solid line indicates all galaxies of type Sa and Sab, the dashed line indicates the S0's.

surface density drops below a value of $1 M_{\odot} \mathrm{pc}^{-2}$ (equivalent to $1.25 \times 10^{20}$ atoms $\mathrm{cm}^{-2}$ ). The derived radii are given in Table 3 .

Finally, we derived the average HI surface density within the HI radius $\left(\left\langle\Sigma_{\mathrm{HI}}\right\rangle_{R_{\mathrm{HI}}}\right)$, as well as within the optical radius $\left(\left\langle\Sigma_{\mathrm{HI}}\right\rangle_{R_{25}}\right)$. The optical radii were derived from the absorption corrected 25 th $B$-band mag $\operatorname{arcsec}^{-2}$ diameters, taken from LEDA. The average HI surface densities are also listed in Table 3.

\section{Hı properties of early-type disk galaxies}

As mentioned in the introduction, the data presented here are intended for a study of rotation curves and dark matter in earlytype disk galaxies. In this paper, however, we focus on the global HI properties of our sample galaxies, and the relation with their optical characteristics; several aspects of this analysis are discussed below.

\subsection{Hı content}

In Fig. 2, we show the distribution of $M_{\mathrm{HI}} / L_{B}$. For $L_{B}$, we used the absolute $B$-band magnitudes from LEDA (column (6) in Table 1) and a solar absolute magnitude in the B-band of 5.47 (Cox 2000). For comparison, we have split the sample in $\mathrm{SO} / \mathrm{SOa}$ 's and Sa/Sab's.

The average values of $\log \left(M_{\mathrm{HI}} / L_{B}\right)$ for $\mathrm{Sa} / \mathrm{Sab}$ 's and S0/S0a's is $-0.62 \pm 0.44$ and $-0.50 \pm 0.40$ in solar units respectively, where the errors give the standard deviations of the respective distributions. These values are higher than the average values for early-type disks found by Roberts \& Haynes (1994), confirming that we have predominantly selected gasrich galaxies in our sample (cf. Sect. 2 and Fig. 1). There is, however, a large variation in the HI content of our galaxies; the

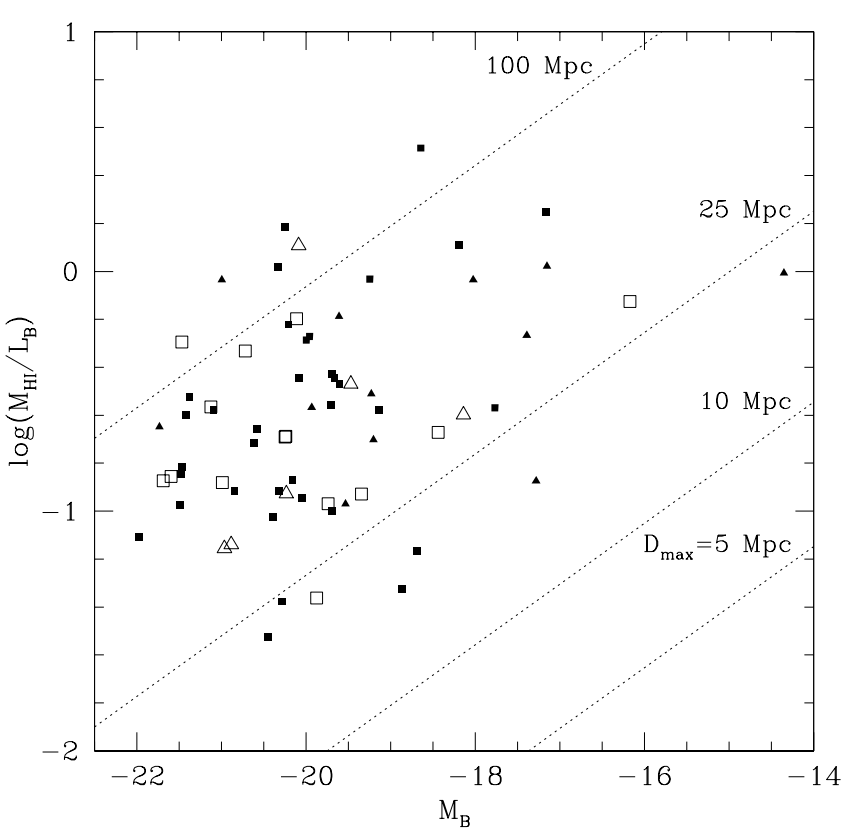

Fig. 3. $M_{\mathrm{HI}} / L_{B}$ versus absolute $B$-band magnitude. $\mathrm{Sa} / \mathrm{Sab}$ galaxies are indicated by squares, $\mathrm{S} 0 / \mathrm{S} 0 \mathrm{a}$ 's by triangles. The filled symbols indicate galaxies whose morphology is not or only mildly asymmetric, open symbols denote moderately or strongly lopsided galaxies. The dashed lines indicate the maximum distance at which a galaxy would still be included in our sample (see text).

most gas-rich galaxies contain about 100 times more gas, compared to their stellar luminosity, than the most gas-poor systems. This range is larger than seen in most late-type galaxies, which span at most only one order of magnitude in gas content (Roberts \& Haynes 1994; Broeils \& Rhee 1997; Paper I).

The large spread in HI content is also apparent in Fig. 3, where we plot the HI mass-to-light ratio versus $B$-band luminosity. In later-type galaxies, a general trend exists, such that the more luminous galaxies contain relatively less HI (Verheijen \& Sancisi 2001; Paper I). In our sample, this correlation is virtually absent. There seems to be a lack of lowluminosity galaxies $\left(M_{B} \gtrsim-18.5\right)$ with low relative gas content, but this is probably a selection effect. The dotted lines in Fig. 3 give the maximum distance $D_{\max }$ out to which galaxies would be included in our sample. $D_{\max }$ is defined as the distance where the flux density of a galaxy, given by $f=F_{\mathrm{HI}} / W$, drops below $20 \mathrm{mJy}$. $W$, the profile width, is estimated from the absolute magnitude using the Tully-Fisher relation found by Verheijen (2001) and assuming an average inclination of $60^{\circ}$. It is obvious that high-luminosity galaxies with a low gas content can be observed out to much larger distances than lowluminosity systems. Low-luminosity $\left(M_{B} \gtrsim-18.5\right)$ galaxies with low relative gas content $\left(\log \left(M_{\mathrm{HI}} / L_{B}\right) \lessgtr-1\right)$ may be as common as their high-luminosity counterparts, the lack of lowluminosity, low $M_{\mathrm{HI}} / L_{B}$ galaxies simply being the result of the smaller volume that our survey covers for these systems.

The large spread in gas content could be explained if a substantial fraction of our early-type disk galaxies have recently accreted gas from small dwarf galaxies. The spread in gas content would then reflect the accretion history of these galaxies. 
Indeed, many of our galaxies show signs of recent interaction or accretion (see Table 3), but the degree of asymmetry in the gas distribution does not correlate with the gas fraction, as can be seen in Fig. 3. It seems unlikely that recent accretion or merger events alone can explain the observed spread in gas content of these galaxies.

Another possible explanation for the large spread lies in the fact that HI content is a property of the disk, whereas our galaxies contain an additional bulge component. If indeed bulges contribute to the optical luminosity but are not related to the HI content, variations in bulge-to-disk luminosity ratios would lead to additional scatter in plots like Figs. 2 and 3. We have checked this hypothesis for a number of galaxies in our sample for which we have accurate optical photometry and bulge-disk decompositions at our disposal, but found that the scatter in gas content did not decrease significantly when, instead of the total luminosity, we only considered the luminosity of the disk component.

Thus, the HI content in these early-type disk galaxies seems to be rather independent of any basic optical property. In Sect. 4.3, we will show that the HI content is correlated with the relative extent of the gas disks, in the sense that the gas disks of gas-rich galaxies are more extended, but of similar surface density, than those of gas-poor systems. Although this implies that the large spread in gas content is, at least partly, related to the large range in the relative sizes of the gas disks (cf. Sect. 4.2), it still offers no explanation as to why some galaxies have larger gas disks, and correspondingly larger HI masses, than others. The origin of the large spread in gas content in these systems remains therefore puzzling.

\subsection{Hı diameters}

In Fig. 4, we show the distribution of $D_{\mathrm{HI}} / D_{25}^{B, c}$, with $D_{\mathrm{HI}}$ the diameters of the HI disks, measured at the $1 M_{\odot} \mathrm{pc}^{-2}$ level. $D_{25}^{B, c}$ are the optical diameters, measured at the absorption corrected 25 th $B$-band mag $\operatorname{arcsec}^{-2}$ level, taken from LEDA. The average ratio of $\mathrm{HI}$ to optical diameter for the $49 \mathrm{Sa} / \mathrm{Sab}$ galaxies in our sample is 1.72 \pm 0.70 ; as above, the error gives the standard deviation of the distribution. This average value is identical, but with larger spread, to the value found by Broeils \& Rhee (1997) for a sample of 108 spiral galaxies, mostly of intermediate and late type.

$9 \mathrm{Sa} / \mathrm{Sab}$ galaxies $(18 \%)$ in our sample have $D_{\mathrm{HI}}<D_{25}^{B, c}$. Most of these cases show clear signs of interaction (e.g. UGC $4862,5559,6621)$ and it seems likely that these galaxies have lost part of their gas disks in the encounters. This would be consistent with the results of Cayatte et al. (1994), who showed that galaxies in the center of the Virgo cluster have smaller HI disks than those in the field and argued that this is caused by ram-pressure stripping and interactions between the cluster members. However, some galaxies with small gas disks in our sample seem undisturbed (e.g. UGC 7489 or 11914); the origin of the small extent of their HI disks is unclear.

The average ratio of HI to optical diameter for the S0/S0a's in our sample is $2.11 \pm 0.70$. This is slightly higher than for the $\mathrm{Sa} / \mathrm{Sab}$ 's, but the number of objects is too small to be able to

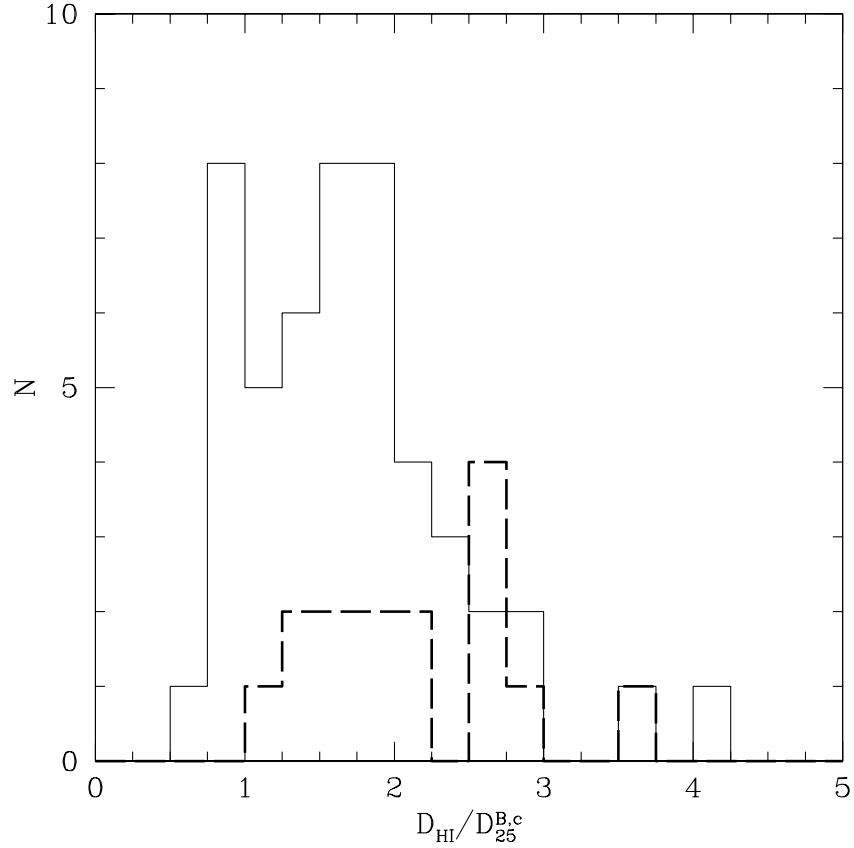

Fig. 4. The distribution of $D_{\mathrm{HI}} / D_{25}^{B, c}$. The solid line indicates all galaxies with type Sa and Sab, the dashed line indicates the S0's.

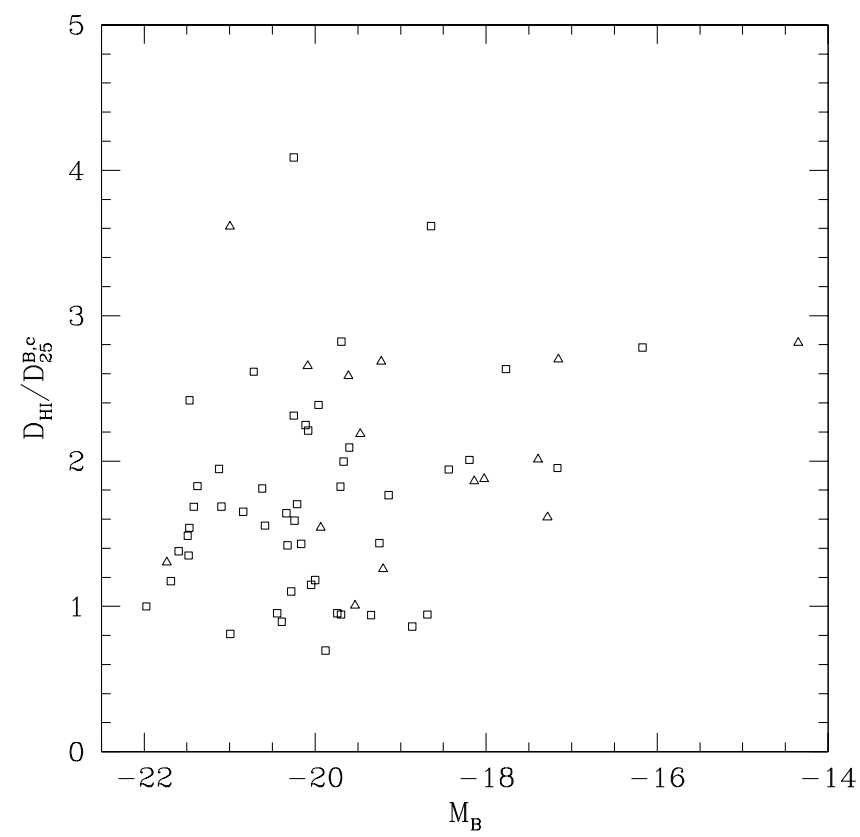

Fig. 5. Ratio of HI to optical diameter $D_{\mathrm{HI}} / D_{25}^{B, c}$ versus absolute $B$ band magnitude. $\mathrm{Sa} / \mathrm{Sab}$ galaxies are indicated by squares, $\mathrm{S} 0 / \mathrm{S} 0 \mathrm{a}$ 's by triangles.

say whether this is significant. Note that three S0/S0a galaxies in our sample (UGC 2154, 3426 and 4637) have an azimuthally averaged HI surface density less than $1 M_{\odot} \mathrm{pc}^{-2}$ everywhere, such that their HI radius is not defined; the gas in these three galaxies is clearly distorted and probably originates from a recent merger or interaction event.

The ratio $D_{\mathrm{HI}} / D_{25}^{B, c}$ between $\mathrm{HI}$ and optical diameters shows no clear correlation with optical luminosity (Fig. 5). The lack of low-luminosity galaxies with small HI disks is probably the 


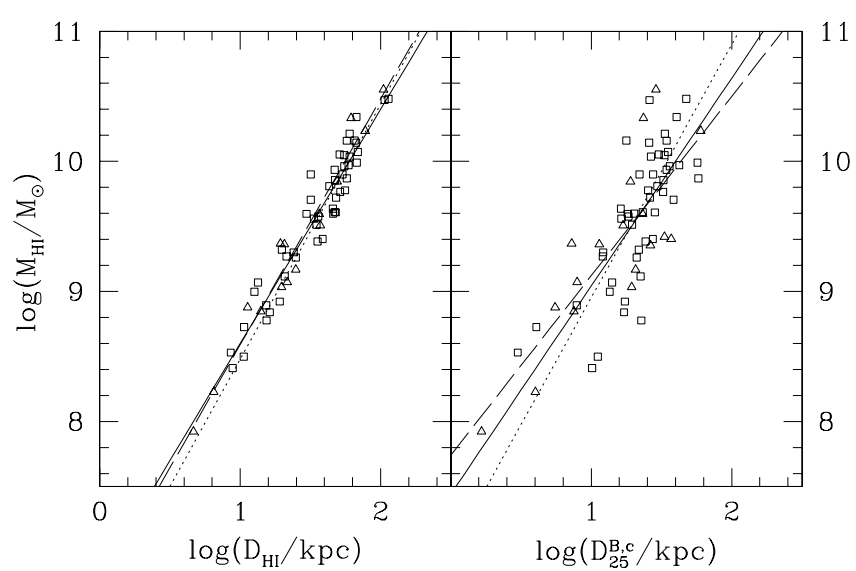

Fig. 6. Total HI mass versus HI (left) and optical (right) diameter. $\mathrm{Sa} / \mathrm{Sab}$ galaxies are indicated by squares, $\mathrm{S} 0 / \mathrm{S} 0$ a's by triangles; the solid and long-dashed lines give the corresponding fits. The dotted lines give the relations found by Broeils \& Rhee (1997).

result of the same selection effects as described in the previous section.

\subsection{Hi surface brightness}

Previous studies have revealed a tight relation between the total HI mass and HI diameter of a galaxy, with $M_{\mathrm{HI}} \propto\left(D_{\mathrm{HI}}\right)^{\alpha}$, $\alpha \approx 1.9$ (Broeils \& Rhee 1997; Verheijen \& Sancisi 2001; Paper I). The small scatter around this relation, and the fact that the slope is so close to 2, imply that the average HI surface density within the $1 M_{\odot} \mathrm{pc}^{-2}$ isophote is almost universal from galaxy to galaxy. The correlation of HI mass with optical diameter is well defined too, but with larger scatter.

These relations are also present in the early-type galaxies studied here, as can be seen in Fig. 6. The relation between $M_{\mathrm{HI}}$ and $D_{\mathrm{HI}}$ is indistinguishable from the one found in previous studies (the dotted line shows the relation from Broeils \& Rhee 1997). We find:

$$
\begin{aligned}
& \log \left(M_{\mathrm{HI}} / M_{\odot}\right)=1.80 \log \left(D_{\mathrm{HI}} / \mathrm{kpc}\right)+6.80 \\
& \log \left(M_{\mathrm{HI}} / M_{\odot}\right)=1.89 \log \left(D_{\mathrm{HI}} / \mathrm{kpc}\right)+6.70
\end{aligned}
$$

The scatter around each of the fits is 0.15 and 0.14 dex respectively, comparable to the dispersion found by Broeils \& Rhee (1997).

We find a slightly shallower relation between HI mass and optical diameter:

$$
\begin{aligned}
& \log \left(M_{\mathrm{HI}} / M_{\odot}\right)=1.60 \log \left(D_{25}^{B, c} / \mathrm{kpc}\right)+7.44 \\
& \log \left(M_{\mathrm{HI}} / M_{\odot}\right)=1.38 \log \left(D_{25}^{B, c} / \mathrm{kpc}\right)+7.74 \\
& \text { (SO/SOa). }
\end{aligned}
$$

The shallow slopes in these relations could again be the result of a selection bias in our survey. Small, low HI-mass galaxies are unlikely to be included in our sample, which explains why the galaxies in our sample with $D_{25}^{B, c}<10 \mathrm{kpc}$ lie systematically above the relation found by Broeils \& Rhee (1997). Given our selection criteria, we see no reason to conclude that early-type disk galaxies follow a different $M_{\mathrm{HI}}-D_{25}^{B, c}$ relation than latertype spirals. Note however that the scatter, 0.34 and 0.38 dex for the $\mathrm{Sa} / \mathrm{Sab}$ and $\mathrm{S} 0 / \mathrm{S} 0$ a samples respectively, is larger than the one found by Broeils \& Rhee (1997).

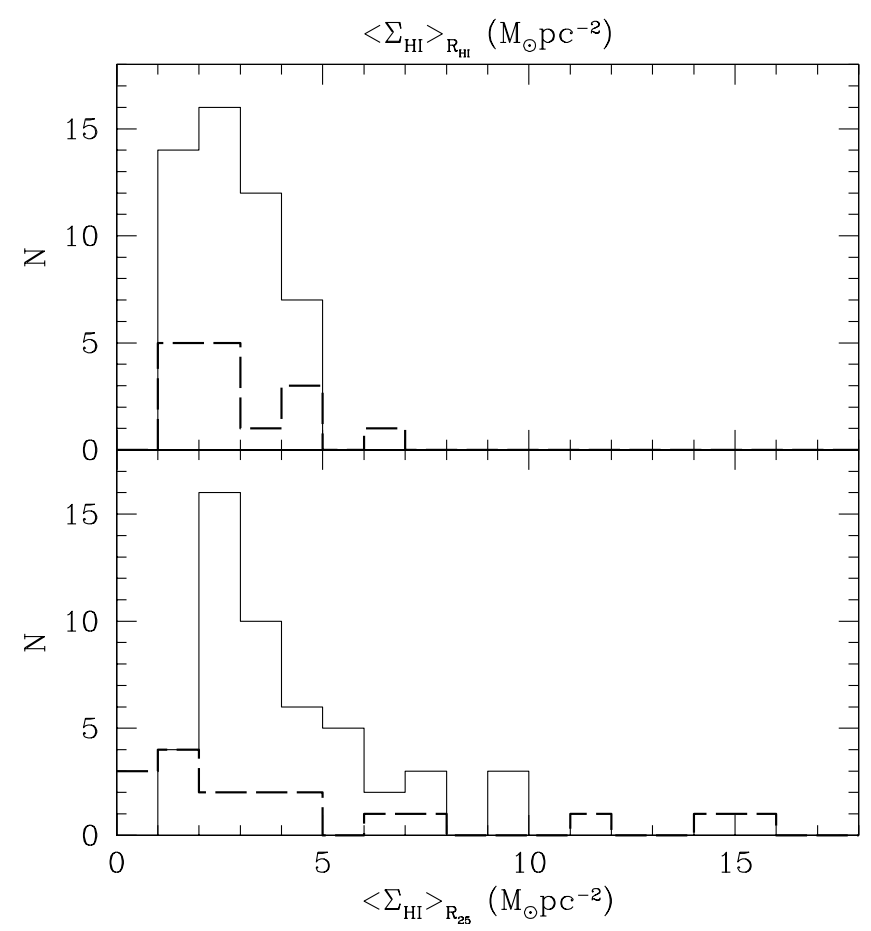

Fig. 7. The distribution of average HI surface density within the $1 M_{\odot} \mathrm{pc}^{-2}$ isophote (top) and within the optical diameter (bottom). The solid line indicates all galaxies with type $\mathrm{Sa}$ and $\mathrm{Sab}$, the dashed line indicates the S0's.

In Fig. 7, we show the distributions of average HI surface density within the HI and optical diameters.

The small scatter in the $M_{\mathrm{HI}}-D_{\mathrm{HI}}$ relation translates into a narrow distribution in $\left\langle\Sigma_{\mathrm{HI}}\right\rangle_{R_{\mathrm{HI}}}$. The average values of $\left\langle\Sigma_{\mathrm{HI}}\right\rangle_{R_{\mathrm{HI}}}$ are $2.8 \pm 0.8$ and $2.9 \pm 1.4 M_{\odot} \mathrm{pc}^{-2}$ for $\mathrm{Sa} / \mathrm{Sab}$ and $\mathrm{S} 0 / \mathrm{S} 0 \mathrm{a}$ galaxies respectively; the errors give the standard deviations of the distributions. Thus, early-type disk galaxies have on average somewhat lower HI surface densities than later-type spirals, but with similar scatter (Broeils \& Rhee 1997). The dispersion in the distribution of $\left\langle\Sigma_{\mathrm{HI}}\right\rangle_{R_{25}}$ is much larger, but the average values are again lower than in later-type spirals (cf. Roberts \& Haynes 1994; Broeils \& Rhee 1997).

As a last illustration that the average HI surface density shows little variation from galaxy to galaxy, we show in Fig. 8 the relation between relative gas content and the HI-to-optical diameter ratio.

This figure shows that if a galaxy has a relatively high gas content, it is because its gas disk is relatively extended; thus the average HI surface density will still be comparable to that in more gas-poor systems. It is, however, still unclear why some galaxies have much larger HI disks than others (i.e. are much more gas-rich, cf. Sects. 4.1 and 4.2).

\subsection{Holes, rings and the relation with star formation}

Most early-type disk galaxies presented here whose HI is concentrated in a regular disk, have a central hole or depression in their HI distribution. Galaxies where we do not observe a central hole are either not well resolved, or do not have a regularly rotating disk; it is likely therefore, that all early-type galaxies 


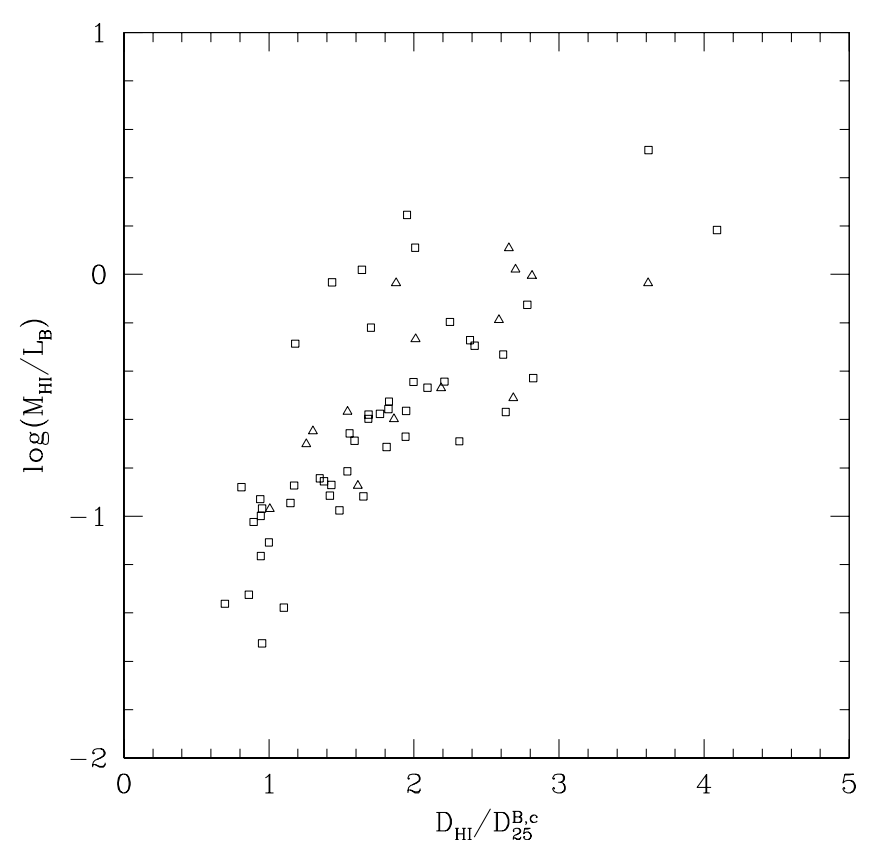

Fig. 8. Relative gas content, $M_{\mathrm{HI}} / L_{B}$, versus relative gas diameter, $D_{\mathrm{HI}} / D_{25}^{B, c} . \mathrm{Sa} / \mathrm{Sab}$ galaxies are indicated by squares, S0/S0a's by triangles.

with a regular gas disk have a central depression in their HI distribution.

In some galaxies, most of the gas is concentrated in rings. In most cases, the rings follow the orientation of the optical disks, but there are also cases (e.g. UGC 12276) where the orientation of the ring is different from that inferred from the optical image or the velocity field. Galaxies with gas rings offer an interesting possibility to study the relation between neutral gas and the stellar light distribution. In Fig. 9 we compare the radial gas distribution for 6 galaxies with distinct gas rings with their $B$-band luminosity profile. The gas surface density profiles are derived from the HI profiles shown in Appendix A, multiplied by 1.44 to account for the presence of other elements (mostly helium). The photometric profiles are taken from a parallel study of the stellar mass distribution in a subsample of the galaxies presented here (Noordermeer et al., in prep.).

UGC 1541 and 3546 (top panels) are barred galaxies with distinct spiral arms. In both cases there is a slight overdensity of blue light at the same radius as the gas ring; this may simply reflect the fact that gas and stars respond in the same way to the gravitational perturbations from the bar and spiral arms.

The other four galaxies, however, are not barred, and have very little spiral structure. Yet in two cases, UGC 3993 and 11914 (middle panels), the HI surface density peaks correspond to small but distinct overdensities of light at the same radii. In UGC 11914 this excess light is related to a prominent ring of blue stars in the original images (see also Buta et al. 1995). $\mathrm{H} \alpha$ emission is detected in this ring as well (Pogge 1989), indicating that the gas is actively forming stars (Battinelli et al. 2000). We are not aware of $\mathrm{H} \alpha$ observations of UGC 3993, but the stars in its ring are significantly bluer than in the central parts, indicating that a population of young stars may exist in this ring as well. In contrast, in UGC 2487 and 6787 (bottom panels), no excess light over the regular exponential disks is detected and the colors of the stars do not vary over the ring. Pogge \& Eskridge (1993) found no H $\alpha$ emission in UGC 2487.

In an attempt to understand why there is no current star formation in the rings of UGC 2487 and 6787, while there are strong indications that it is happening in UGC 3993 and 11914 where the total gas densities are similar, we compared the observed gas densities to the threshold density for star formation derived by Kennicutt (1989). Assuming that star formation in spiral galaxies is regulated by the onset of gravitational instabilities in their gas disks, Kennicutt applied the criterion for disk stability from Toomre (1964) to these systems and derived the following threshold density for star formation:

$\Sigma_{\text {crit }}=\alpha \frac{\kappa c}{3.36 G}$.

$\kappa$ is the epicyclic frequency, which we derived from rotation curves from tilted ring fits to the velocity fields. For $c$, the velocity dispersion of the gas, we followed Kennicutt, and assumed a constant value of $6 \mathrm{~km} \mathrm{~s}^{-1}$. For the high surfacebrightness galaxies studied here, this value is probably on the low side, and the actual values of $\Sigma_{\text {crit }}$ may be higher than what we derive here. However, higher values for the threshold density will only aggravate the discrepancy described below, and we choose $c=6 \mathrm{~km} \mathrm{~s}^{-1}$ to be able to compare our results directly with Kennicutt. $\alpha$ is a dimensionless quantity of order unity; from an empirical study of star formation cutoffs in spiral galaxies, Kennicutt derived a value of $\alpha=0.67$. The applicability of this recipe was recently confirmed by Martin \& Kennicutt (2001) for a sample of 32 nearby spiral galaxies. The critical densities in our galaxies are plotted, as a function of radius, with the dashed lines in Fig. 9. Due to the large rotation velocities and the correspondingly large $\kappa$, the critical densities are high and the observed gas densities are far below the threshold in all cases.

Thus, if the description of star formation thresholds by Kennicutt (1989) is correct, it is not surprising that we do not see a correlation between the HI rings and the stellar light distribution in UGC 2487 and 6787; the gas densities are simply much too low to sustain large-scale star formation. But then it is unclear why UGC 3993 and 11914 do form stars. In fact, the galaxy out of these 6 which has the highest star formation activity, UGC 11914 , has the lowest ratio $(<0.25$ everywhere) of observed to critical density!

Note that we have ignored the contribution of molecular gas to the total gas densities. It seems, however, unlikely that the presence of molecular gas can explain the discrepancy between observed and critical gas densities in these galaxies. Several $\mathrm{CO}$-surveys have been carried out in recent years, and all find that molecular gas is concentrated in the inner parts of galaxies (Sofue et al. 1995; Wong \& Blitz 2002; Sofue et al. 2003), with exponential scale lengths comparable to those of the underlying stellar disks (Regan et al. 2001). No substantial column densities of CO emission are generally observed at large radii. Thus, while we may have underestimated the total gas densities in the inner regions of the galaxies in Fig. 9, the effect will be small in the HI rings of interest, which all lie at large radii, and the total gas densities are truly smaller than the critical density. 

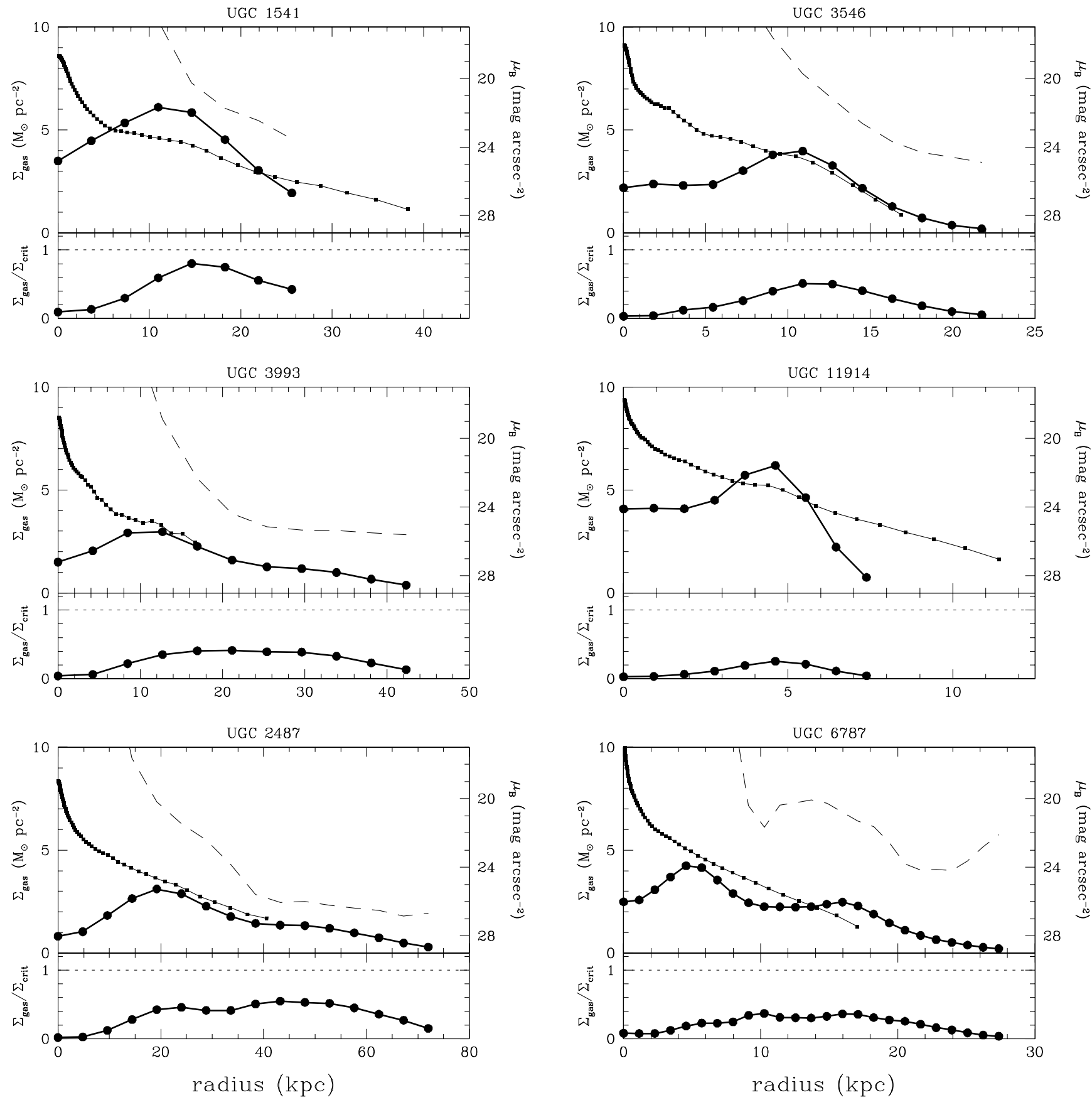

Fig. 9. Comparison between gas and stellar light distributions in galaxies with pronounced gas rings. UGC 1541 and 3546 are barred galaxies; the others have no bars. Top panels: filled circles and bold lines show the HI surface density profiles, multiplied by 1.44 to account for the presence of helium. Radii are converted to kpc. Filled squares and thin lines show B-band photometric profiles. Dashed lines show the critical density for star formation, according to Kennicutt (1989). For UGC 11914, the critical density is larger than $10 M_{\odot} \mathrm{pc}^{-2} \mathrm{everywhere.} \mathrm{Bottom}$ panels: ratio between observed gas density and critical density. The dotted line indicates the threshold for star formation.

In recent years, more cases have been reported of star formation in regions where the gas density is lower than the critical density from Eq. (5). Kennicutt (1989) and Martin \& Kennicutt (2001) already noted that a fraction of the galaxies in their sample (notably M33 and NGC 2043) showed widespread star formation, even though their gas surface densities were below the threshold. van Zee et al. (1997) observed 11 dwarf galaxies and showed that, although the gas densities were below the threshold in all cases, some of them had a substantial level of star formation. Several recent papers report the detection of star formation in the outskirts of nearby galaxies, where the observed gas densities are well below the threshold densities for star formation too (Ferguson et al. 1998; Cuillandre et al. 2001; Thilker et al. 2005). Thus, a number of cases have now been identified where the simple criterion of Kennicutt (1989) does not apply. 
Recently, Schaye (2004) argued that, rather than being regulated by global, gravitational instabilities, star formation requires a phase transition from warm to cold gas, and is thus governed by thermal instabilities instead. He predicted that the critical density for star formation is more or less independent of global properties such as rotation velocity, and has a universal value in the range 3-10 $M_{\odot} \mathrm{pc}^{-2}$. Although it is not clear how this prediction compares to the large spread in observed threshold densities from Kennicutt (1989), it is suggestive that all galaxies in Fig. 9 have gas densities around the lower end of this range, and would thus be at the verge of instability for star formation.

It seems, however, premature to completely abandon Kennicutt's theory, until more knowledge is obtained about the small-scale distribution of the gas (cf. van Zee et al. 1997). Braun (1997) studied the structure of HI disks in 11 wellresolved, nearby galaxies and found that the HI was distributed in small, high-density regions with covering factors between 6 and 50\%. Thus, if UGC 3993 and 11914 are extreme cases where the covering factor is low, and UGC 2487 and 6787 have a smoother HI distribution, the observed (lack of) correlation between HI distribution and star formation in these galaxies might still be consistent with the predictions from Kennicutt (1989). Note, however, that Kennicutt did not explicitly account for the "patchiness" of gas in his galaxies; an average amount of substructure in the gas distribution must implicitly be included in his parameter $\alpha$. There is no a priori reason to assume that galaxies such as UGC 3993 or 11914 have much lower covering factors than average, but we cannot rule out this possibility, and high-resolution observations are needed to check this.

\subsection{Lopsidedness}

Asymmetries in the morphology and kinematics of galaxies are still a subject of debate. Rix \& Zaritsky (1995) found that about one third of all spiral galaxies show large-scale asymmetries in their optical images. Global HI-profiles have asymmetric shapes for at least 50\% of all disk galaxies (Baldwin et al. 1980; Richter \& Sancisi 1994; Haynes et al. 1998), but without a full 2D mapping of the HI component in these galaxies, it is difficult to determine the origin of the asymmetries in the profiles. With the advent of a large number of spatially resolved $\mathrm{HI}$ images and velocity fields, it was found that not only the spatial distribution of the gas is often asymmetric, but that many galaxies are also lopsided in their kinematics: Swaters et al. (1999) estimated that about half of all disk galaxies have asymmetric kinematics. Lopsidedness seems particularly common in late-type spirals; Matthews et al. (1998) found that out of a sample of 30 extremely late-type galaxies, about $75 \%$ had clearly lopsided HI profiles.

In some cases, lopsidedness can be linked to ongoing interactions with companion galaxies. However, lopsidedness is also common in isolated galaxies (Wilcots \& Prescott 2004), implying that lopsidedness may be an intrinsic feature of disk galaxies and that the underlying dark matter distribution may be asymmetric too (Jog 1997; Noordermeer et al. 2001). Even if lopsidedness in galaxies is triggered by tidal interactions or mergers, it must be a long-lived phenomenon to explain the relatively high frequency of lopsidedness in isolated systems.

A full, quantitative analysis of the occurrence of lopsidedness in the galaxies in our sample is beyond the scope of the present paper. Instead, we have determined by eye how many of our early-type galaxies are lopsided, and briefly discuss the results here. The degrees of asymmetry in the global profile, morphological appearance and kinematical structure (velocity field and major axis $x v$-diagram) are indicated with stars in Table 3, where $0,1,2$ or 3 stars mean not, mildly, moderately or severely lopsided respectively. In some cases the gas is too strongly distorted or too poorly resolved to determine whether it is asymmetric. These cases are indicated with n.a. (not available).

Asymmetries in the global profiles and gas distribution are very common in the early-type disk galaxies studied here. Respectively 35 and 34 galaxies (51 and 50\%) have at least mildly asymmetric global profiles or surface density maps. In most cases, however, the asymmetries can be related to the presence of or interactions with companion galaxies. All galaxies, except one (UGC 5060), which have severely lopsided gas distributions, and most cases where the gas distribution is moderately lopsided, show clear signs of ongoing interactions. On the other hand, morphological lopsidedness is rare in early-type disk galaxies without nearby companions or signs of recent interactions. Of all 41 galaxies that do not show clear signs of interaction, only 15 (37\%) are morphologically asymmetric, usually only mildly. Only 3 isolated galaxies (7\%) are more than mildly asymmetric.

Kinematical lopsidedness is rare in early-type galaxies in general, interacting or not. Out of all the galaxies for which we can determine the degree of symmetry in the kinematics (53), UGC 624 is the only one with a severely lopsided velocity field; in this case the lopsidedness can be explained as a result of tidal interaction with its nearby companion UGC 623 . The only two cases of isolated galaxies with significantly lopsided kinematics, UGC 5960 and UGC 11852, are both extreme galaxies in our sample in the sense that the former is one of the least luminous galaxies in our sample $\left(M_{B}=-17.39\right)$, while the latter has the most extended gas disk, relative to the optical diameter, of all our sample galaxies $\left(D_{\mathrm{HI}} / D_{25}^{B, c}=4.1\right)$.

We conclude that lopsidedness in the HI distribution is at least as common in early-type disk galaxies as in later-type galaxies; truly symmetric gas disks are rare. In most cases, however, morphological asymmetries are clearly related to ongoing interactions or accretion. Morphological lopsidedness in isolated early-type disks, and kinematical asymmetries, are much rarer, supporting the findings of Matthews et al. (1998).

\section{Notes on individual galaxies}

Many galaxies in our sample show interesting features in their HI distribution or kinematics which deserve special emphasis. We discuss these below.

UGC 89 (NGC 23) and UGC 94 (NGC 26) are separated by only $10^{\prime}$. They form a loose group together with Scd galaxy UGC $79\left(20^{\prime}\right.$ to the southwest) and 4 other galaxies 
UGC 89
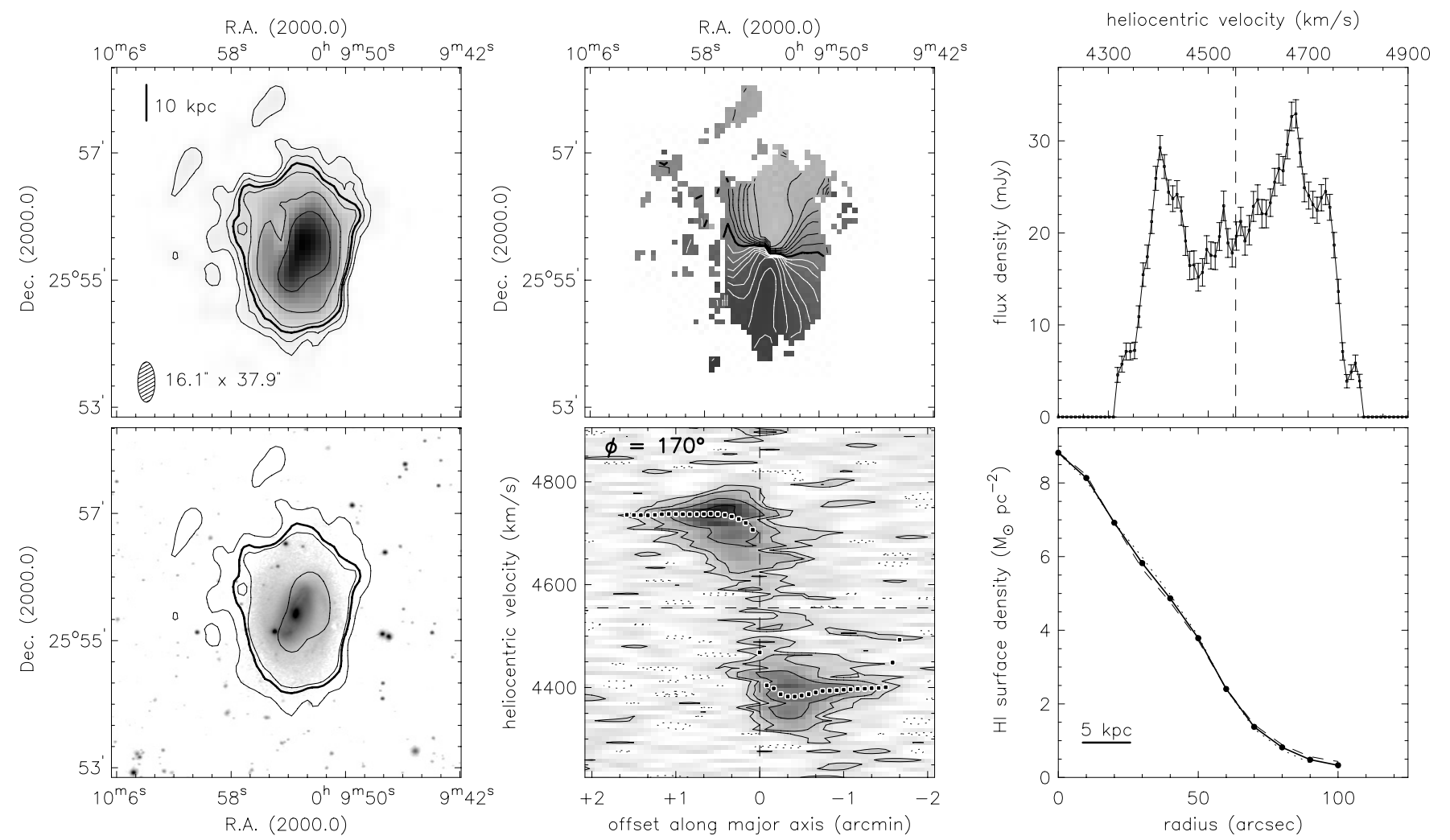

UGC 94
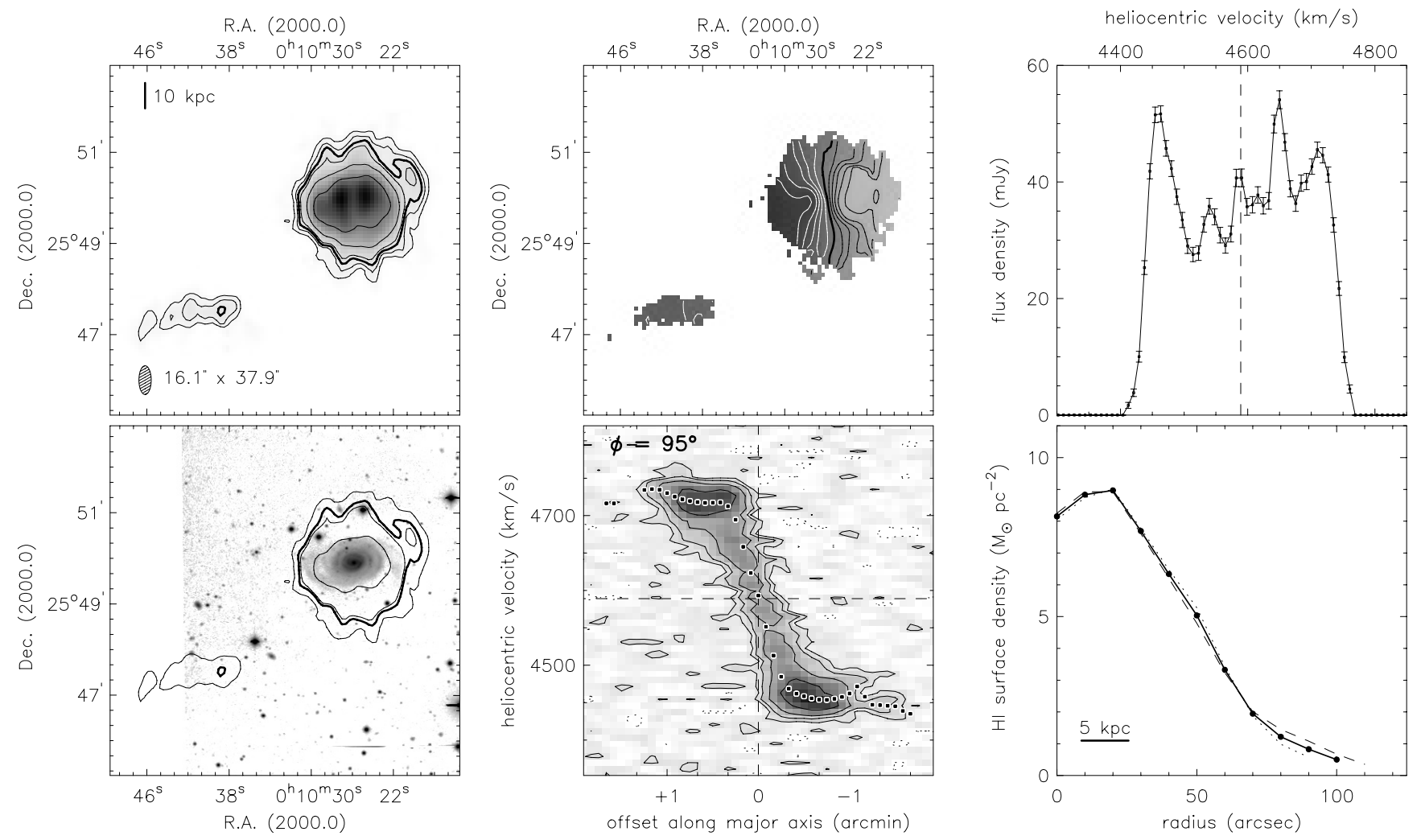

Fig. 10. Example entries from the atlas for UGC 89 and 94 . For each galaxy, the upper left panel shows the integrated HI surface density map. The lower left panels show the same images overlayed on an $R$-band image. The upper middle panels show the velocity fields and the lower middle panels show position-velocity diagrams along the major axis. The upper right panels show the global HI profiles and the lower right panels show the radial surface density profiles. A full description of these figures is given in the electronic version of the article. 
(Garcia 1993). A few small HI clouds are visible in the neighbourhood. Optical counterparts are visible on the DSS for most of them, so they are probably dwarf companions of the main group members. A tidal tail-like structure is visible south-east of UGC 94, indicating a recent interaction or merger event. In our low-resolution data cube, this tidal feature is seen to be connected to the main disk of UGC 94 and extends about 10' $(\sim 180 \mathrm{kpc})$ to the east.

UGC 499 (NGC 262), also known as Markarian 348, is a well-known Seyfert 2 galaxy (Koski 1978). We detect HI emission out to distances of $100 \mathrm{kpc}$ from the center, but it is clearly distorted and does not rotate regularly around the center. UGC 508 (NGC 266) lies about $23^{\prime}$ to the northeast of UGC 499; the peculiar structure of the gas in UGC 499 might be a result of a past interaction with its neighbour. The HI properties of UGC 499 were discussed in detail by Heckman et al. (1982) and Simkin et al. (1987).

UGC 624 (NGC 338) is one of the most lopsided galaxies in our sample, both in the gas distribution and in the kinematics. The asymmetry could be a result of tidal interaction with nearby neighbour UGC 623 . Note also the small "blob" of gas just southeast of the main gas disk.

UGC 1310 (NGC 694) resides in a small group of galaxies, the main other members of which are NGC 680, 691 and 697 (Garcia 1993). The central regions of this group were imaged in HI before by van Moorsel (1988). Apart from UGC 1310, we also detect HI emission in NGC 691, 697 and UGC 1313, but as these are all of later morphological type, they are not included in the present study. The HI disk of UGC 1310 is barely resolved in our observations. As a result, the line profiles are strongly affected by beam-smearing; the method described in Sect. 3.5 to derive the velocity field is therefore inadequate and fails to recover the projected rotational velocities of the gas.

Most of the gas in UGC 2045 (NGC 972) seems to be concentrated in a regularly rotating disk that has the same orientation as dustlanes in the optical image. The line-profiles in this disk have complicated shapes and the method described in Sect. 3.5 for the derivation of the velocity field fails to recover the projected rotational velocities of the gas. In the outer parts, several large filaments of gas are detected; the gas in these filaments clearly does not follow the rotation of the inner parts.

UGC 2154 (NGC 1023) is the brightest galaxy of a nearby group of 13 galaxies (Tully 1980). The total HI-flux for this galaxy is quite large $\left(80.13 \mathrm{Jy} \mathrm{km} \mathrm{s}{ }^{-1}\right)$, but the gas is scattered over a large area, has a very low column-density and does not reside in a regular disk. It seems most likely the result of a recent merger or accretion event, possibly with one of the other group members (cf. Sancisi et al. 1984).

Most of the gas in UGC 2183 (NGC 1056) is concentrated in an edge-on disk which coincides with the dust lane seen in the optical image. Due to this edge-on orientation, projection effects lead to strongly non-Gaussian line profiles; the method described in Sect. 3.5 to derive the velocity field is therefore inadequate and fails to recover the projected rotational velocities of the gas in the central parts. At larger radii, the gas seems to warp out of the plane and the orientation becomes more face-on.
UGC 2487 (NGC 1167) is a nice example of an S0 galaxy with an extended and regularly rotating HI disk. The gas seems to be in circular motion at radii up till $80 \mathrm{kpc}$. The central hole does not reflect a true absence of gas, but is rather the result of absorption against a central continuum source.

The companion east of UGC 2916 is PGC 14370. When the data are smoothed to lower resolution, some emission is seen to bridge the space between the two galaxies, indicating that some interaction is going on. There are clear signs of off-planar gas in PGC 14370, but the main disk of UGC 2916 seems relatively undisturbed.

UGC 2953 (IC 356) is by far the best resolved galaxy in our sample. The gas is concentrated in pronounced spiral arms that extend far beyond the bright optical disk. The velocity field shows distinctive "wiggles" in the isovelocity contours where the gas crosses the arms.

The HI distribution, velocity field and global profile of UGC 3205 are almost perfectly symmetric, except for the twisting of the isovelocity contours in the bar region.

UGC 3354 is almost perfectly edge-on and has a peculiar double warp. Both the stellar and gas disks seem to warp "clockwise" first. At larger radii, where no starlight can be detected, the gas disk reverses its warp to the opposite direction. Projection effects lead to complicated line-profiles in the inner parts of the disk; the method described in Sect. 3.5 to derive the velocity field is inadequate there and fails to recover the projected rotational velocities of the gas.

The gas distribution in UGC $\mathbf{3 4 2 6}$ is irregular and seems to be connected to the gas disk of UGC $3422, \approx 100 \mathrm{kpc}$ to the northwest. The projected surface density of the gas is very low, with a maximum of only about $0.3 M_{\odot} \mathrm{pc}^{-2}$. It appears as if the gas in UGC 3426 has been tidally drawn out of the gas disk of UGC 3422.

UGC 3642 is a very peculiar galaxy. The gas inside the bright optical disk seems to be regularly rotating in the same plane as defined by the stellar light distribution. But further out, the direction of motion is reversed. Each component individually appears undisturbed and the gas seems to be in regular rotation in both the inner and the outer regions. Two options seem possible to explain the peculiar kinematics of this galaxy. The first is that both components are fully decoupled, in which case this galaxy would carry some resemblance with the "Evil Eye" galaxy (NGC 4826), which also has two decoupled gas disks (Braun et al. 1992; Rubin 1994). However, both the total HI mass and the size of the disks in UGC $\mathbf{3 6 4 2}$ are an order of magnitude larger than in NGC 4826. Alternatively, the gas disk could be extremely warped, such that the direction of motion along the line of sight is reversed. We are unable to distinguish between the two options, and more detailed observations and modelling are needed to clarify the nature of this galaxy. Note that the total extent of the outer gas disk is extremely large, with $D_{\mathrm{HI}}>100 \mathrm{kpc}$.

At first sight, UGC 3965 (IC 2204) seems a similar case as UGC 3642, with the kinematical position angle changing by almost $180^{\circ}$. But in this case, the observed velocity field can also be explained by an almost face-on disk with a mild warp. While the gas would be moving in the same direction 
everywhere, its radial velocity along the line of sight could easily be reversed.

UGC 4605 (NGC 2654) is almost perfectly edge-on in the inner parts, but the gas seems mildly warped in the outer parts. The position-velocity diagram shows that the rotation velocities decline strongly towards the edge of the HI disk; the decline starts already before the onset of the warp and mustreflect a true decrease in the rotation velocities. In the very inner parts, the line-profiles are strongly affected by projection effects and the method described in Sect. 3.5 to derive the velocity field fails to recover the projected rotational velocities of the gas there.

UGC 4637 (NGC 2655) is a nearby Seyfert 2 galaxy whose nuclear regions show complex structure, both in high-resolution radio continuum observations as in optical emission lines (Keel \& Hummel 1988). These authors attribute the complexity of the central parts to a recent interaction or merger event. This interpretation seems to be confirmed by the large-scale structure of the neutral gas seen in our observations. The HI is clearly disturbed, with a large extension to the northwest; the gas does seem to have a general sense of rotation, but it is clearly not on regular circular orbits. The optical image of this galaxy shows some distinct loops and shells, further confirming the hypothesis of a recent interaction.

UGC 4666 (NGC 2685) is also known as the Spindle or Helix galaxy, because of its prominent polar ring. Early HI observations of this galaxy were presented by Shane (1980). Our observations, at higher sensitivity and resolution, confirm both the outer gas ring as well as HI related to the helix-like structure inside this ring. The gas in the helix is kinematically distinct from the rest of the galaxy and seen at almost right angles to the outer ring.

UGC 4862 (NGC 2782) has a large, banana-shaped tail of gas northwest of the main disk. The gas in the central regions is clearly disturbed as well and does not show signs of regular rotation around the center. The optical image is peculiar too, with some striking shells. These facts were already noted by Smith (1994), who interpreted the peculiar structure as evidence for a recent merger of the main galaxy with a low-mass companion. Schiminovich et al. (1994, 1995) studied HI in shells around elliptical galaxies. It seems not unreasonable to assume that UGC 4862 is a similar case as their galaxies, but in an earlier stage of its evolution.

The main disk of UGC $\mathbf{5 2 5 3}$ (NGC 2985) is regular in its distribution and kinematics. In the outer parts, a large one-armed spiral of gas is seen to extend towards a small "blob" of gas southwest of the galaxy. UGC 5253 lies about $20^{\prime}$ west of the late-type spiral UGC 5316, which also has a disturbed morphology (e.g. Vorontsov-Vel'Yaminov 1977). The redshifts of the two systems differ by about $250 \mathrm{~km} \mathrm{~s}^{-1}$ only, so the peculiar structures of both galaxies could well be the result of a tidal interaction.

UGC 5559 (NGC 3190) is part of Hickson compact group of galaxies 44, together with UGC 5554, 5556 and 5562 (Hickson et al. 1989). All group members lie in our field of view, but the observations for these galaxies were done with the "old" Westerbork receivers and the sensitivity is insufficient to detect any emission in UGC 5554 and 5562 . UGC 5559 itself is only barely detected, and the signal-to-noise ratio of the data is too low to make a detailed study of the gas distribution in this galaxy. Only for the more gas-rich, late-type spiral UGC 5556 useful HI surface density maps and velocity fields could be obtained, but as this galaxy does not meet our selection criteria, it is not discussed here further.

UGC 5906 (NGC 3380) is poorly resolved and the line-profiles suffer from beam-smearing. As a result, the method described in Sect. 3.5 to derive the velocity field is inadequate and fails to recover the projected rotational velocities of the gas.

UGC 5960 (NGC 3413) is close to edge-on and projection effects lead to non-Gaussian line profiles. As a result, the method described in Sect. 3.5 to derive the velocity field is inadequate and fails to recover the projected rotational velocities of the gas.

Due to the poor resolution and resulting beam-smearing in our observations of UGC $\mathbf{6 0 0 1}$ (NGC 3442), the method described in Sect. 3.5 to derive the velocity field is inadequate and fails to recover the projected rotational velocities of the gas.

The central hole in the disk of UGC 6118 (NGC 3504) is not due to a true absence of gas, but rather an artefact caused by HI absorption against a central continuum source (see also UGC 2487).

UGC 6621 (NGC 3786) and UGC 6623 (NGC 3788) are clearly interacting. A giant tidal tail of HI extends almost $50 \mathrm{kpc}$ northward from UGC 6623. On our optical image, a low-surface-brightness counterpart is visible. Although the gas of both galaxies seems to overlap in the space between them, it is separated in velocity and we can distinguish which gas belongs to which galaxy in the full resolution data cube. Thus we were able to generate radial profiles for the gas in each galaxy separately. At $60^{\prime \prime}$ resolution, the data become too heavily smoothed and the regions of emission from the individual galaxies start overlapping in individual channel maps as well. We cannot make global profiles of the emission of each galaxy separately anymore at this resolution, and we were forced to generate the global profiles from the 30" data. But at 30" already the galaxies are hardly resolved and the masks for this resolution do not miss much flux compared to those of the $60^{\prime \prime}$ data.

UGC 6742 (NGC 3870) is poorly resolved and the line-profiles suffer from beam-smearing. As a result, the method described in Sect. 3.5 to derive the velocity field is inadequate and fails to recover the projected rotational velocities of the gas.

The gas in the inner parts of UGC 6786 (NGC 3900) follows the light distribution of the optical image. Most of the gas in the outer parts is concentrated in two diffuse spiral arms which seem to be warped with respect to the inner parts.

The well-studied galaxy UGC 7166 (NGC 4151) is one of the original Seyfert galaxies (Seyfert 1943). The HI in this galaxy follows the stellar light in the bar and the spiral arms (cf. Pedlar et al. 1992).

UGC 7256 (NGC 4203) has a peculiar, filamentary gas distribution. The gas has a general sense of motion around the center, but it is clearly not on regular circular orbits. This galaxy bears some resemblance with UGC 4637 (see above).

UGC 7489 (NGC 4369) has the smallest HI disk, relative to its optical diameter, of all galaxies in our sample $\left(D_{\mathrm{HI}} / D_{25}^{B, c}=\right.$ $0.86)$, with the exception of a few interacting systems or 
galaxies where the $\mathrm{HI}$ or optical radii are not well defined (e.g. UGC 5559 or 10448). In the optical image, very weak spiral structure and dust absorption can be seen at the locations of the HI emission.

UGC 7704 (NGC 4509) has a peculiar morphology. The optical image has a position angle of about $155^{\circ}$, but the HI map seems to be almost perpendicular to this, with a position angle of about $57^{\circ}$. The kinematical major axis is different again, about $37^{\circ}$. UGC 7704 does not seem to have any major companions which could cause this strange structure, so the true nature of it remains unclear. Due to the poor resolution, the line-profiles in this galaxy suffer from beam-smearing and the method described in Sect. 3.5 to derive the velocity field fails to recover the projected rotational velocities of the gas.

No HI gas is detected in the bulge of UGC 7989 (NGC 4725), nor in the giant bar. It is rather concentrated in narrow spiral arms, which coincide with the stellar arms. The outer arm on the east side is particularly prominent, whereas the arms on the northwest side seem to be truncated, causing marked asymmetries in the HI surface density map and global profile.

Most of the gas in UGC 8271 (NGC 5014) seems to be concentrated in a ring which is tilted with respect to the main stellar disk. Close inspection of the optical image reveals a faint polar ring-like structure, and the $\mathrm{HI}$ at the corresponding locations seems to be rotating in the same plane as defined by this ring. A tail of gas extends further to the south. $12^{\prime}$ to the northnortheast, a number of large HI clouds are detected, all without optical counterparts. UGC 8271 resides in a loose group, the main other members of which are NGC 5005 and 5033. The peculiar phenomena in the gas distribution and kinematics in and around UGC 8271 may all be related and suggest a recent merger or accretion event, possibly with a small gas-rich member of the same group of galaxies. Due to the complicated structure of the gas in this galaxy and the resulting projection effects, the line-profiles are strongly non-Gaussian and the method described in Sect. 3.5 to derive the velocity field fails to recover the projected velocities of the gas.

UGC 8805 (NGC 5347) is at first sight a similar case as UGC 7704. The position angle of the HI-disk seems to be almost perpendicular to that of the optical disk. Note though that we only get sufficient signal at $60^{\prime \prime}$ resolution; the optical disk then fits almost entirely in one resolution element. There is a hint that the $\mathrm{HI}$ is actually elongated along the optical image in the central regions, but observations at higher resolution and sensitivity are required to confirm this. If indeed the gas is aligned with the stars in the inner parts, UGC 8805 could be an example of a galaxy with an extreme warp. This would also explain the strong decline of radial velocities along the major axis, as shown in the $x v$-slice.

The optical image of UGC 8863 (NGC 5377) is completely dominated by a giant bar. Two faint spiral arms are seen to extend from its edges. The HI emission nicely follows the bar and spiral arms; the latter are much more pronounced here. In the center, bar-induced streaming motions produce a twist in the isovelocity contours.

UGC 9133 (NGC 5533) has a large one-armed spiral in the outer regions which seems to be warped with respect to the main disk. The inner parts are highly regular. The $x v$-diagram indicates that the rotation velocities are declining. In the outer parts, this can be explained as a result of the warp, but the decline sets in well within the radius of the warp and must reflect a truly falling rotation curve.

The HI which we detect in UGC 10448 (NGC 6186) is very peculiar. The gas seems offset from the optical image by about $30^{\prime \prime}$. Close inspection of the optical image reveals a faint galaxy behind the main disk. Thus, we are seeing here the coincidental alignment of two spiral galaxies. This is further confirmed by the redshift of the $\mathrm{HI}$ emission of about $11350 \mathrm{~km} \mathrm{~s}^{-1}$, whereas the optical redshift of the foreground galaxy is $2935 \mathrm{~km} \mathrm{~s}^{-1}$ (UZC; Falco et al. 1999). The foreground galaxy has been detected at the correct redshift in $\mathrm{HI}$ as well (Rosenberg \& Schneider 2000), but the UGC contains the redshift of the background system and confused us into observing at the wrong frequency. As no morphological classification, nor photometric data, are available for the background galaxy, we have excluded this galaxy from the statistical analysis in Sect. 4, and merely show the data for general interest.

UGC 11269 (NGC 6667) must recently have undergone a merger. A giant one-armed spiral arm seems to extend out from the main disk. It is partly visible in the optical image as well. Most of this material seems to be virialized already, since it follows closely the velocities of the gas in the inner parts. Note also the peculiar, asymmetric shape of the position-velocity diagram along the major axis: at the receding side, the peak velocity is much higher than at the approaching side. On both sides, the rotation velocities decline strongly away from the center.

Most of the gas in UGC 11670 (NGC 7013) is concentrated in the bar and the spiral arms. In the outer regions, the gas distribution is irregular, with several filaments emanating from the main disk. The line profiles in the inner parts are strongly non-Gaussian; the method described in Sect. 3.5 to derive the velocity field is inadequate there and fails to recover the projected rotational velocities of the gas.

The gas in UGC 11914 (NGC 7217) is predominantly concentrated in a ring. The stellar population in the ring is distinctively bluer than the surroundings, indicating that the gas is associated with an enhanced level of star formation (see Sect. 4.4).

Most of the gas in UGC 12276 (NGC 7440) is concentrated in a ring which is elongated perpendicular to the optical and kinematical major axis. It seems unlikely that this peculiar geometry can be explained solely by the presence of the little companion $\approx 40 \mathrm{kpc}$ to the east, so the true nature of it remains unclear.

Due to the poor resolution and resulting beam-smearing in the observations of UGC 12713, the method described in Sect. 3.5 to derive the velocity field is inadequate and fails to recover the projected rotational velocities of the gas.

UGC 12815 (NGC 7771) is strongly distorted and seems to be interacting with its neighbours UGC 12813, NGC 7771A and UGC 12808. Two giant tidal tails extend to more than $100 \mathrm{kpc}$ away from the system.

\section{Conclusions}

In the previous sections, we have presented the results of a study of the HI properties of a sample of 68 early-type disk 
galaxies, with morphological type ranging from S0 to Sab and absolute $B$-band magnitude between -14 and -22 . A number of conclusions can be drawn:

- The HI content of early-type disk galaxies is highly variable. There is a wide range in HI mass-to-light ratios $M_{\mathrm{HI}} / L_{B}$, with the most $\mathrm{HI}$ rich galaxies in our sample containing about 2 orders of magnitude more gas, relative to the stellar luminosity, than the most gas-poor systems. The average values for $\log \left(M_{\mathrm{HI}} / L_{B}\right)$ are $-0.62 \pm 0.44$ and $-0.50 \pm 0.40$ in solar units for $\mathrm{Sa} / \mathrm{Sab}$ and $\mathrm{S} 0 / \mathrm{S} 0$ a galaxies respectively. The errors give the standard deviations of the distributions.

- The average ratio $D_{\mathrm{HI}} / D_{25}^{B, c}$ between HI and optical diameter, defined at $1 M_{\odot} \mathrm{pc}^{-2}$ and $25 \mathrm{~B}$-band mag $\operatorname{arcsec}^{-2}$ respectively, is $1.72 \pm 0.70$ for $\mathrm{Sa} / \mathrm{Sab}$ galaxies and $2.11 \pm$ 0.70 for $\mathrm{S} 0 / \mathrm{S} 0 \mathrm{a}$ 's. These values are comparable to those observed for later-type spiral galaxies, but with larger spread.

- The average HI surface brightness in our sample galaxies is slightly lower than that in later-type galaxies. Within our sample, the variations from galaxy to galaxy are small. The average values for $\left\langle\Sigma_{\mathrm{HI}}\right\rangle_{R_{\mathrm{HI}}}$, the effective HI surface brightness inside the $1 M_{\odot} \mathrm{pc}^{-2}$ isophote, are $2.8 \pm 0.8$ and $2.9 \pm 1.4$ for $\mathrm{Sa} / \mathrm{Sab}$ and S0/S0a galaxies respectively.

- All early-type galaxies whose gas is distributed in a regular rotating disk have a central hole or depression in their HI distribution.

- A number of galaxies in our sample have distinct, axisymmetric rings of gas. In some of these cases, the overdensity of gas coincides with regions of enhanced star formation and a population of young stars, even though the gas densities are far below the threshold for star formation derived by Kennicutt (1989). In other cases no star formation activity is present, even though the gas densities are comparable. These discrepancies suggest the existence of an additional regulation mechanism for star formation at low gas densities, the exact nature of which still needs to be clarified.

- Morphological and kinematical peculiarities are very common in early-type disk galaxies, often related to ongoing or recent interaction events. In many galaxies, we see indications for a tidal origin of the gas. Interactions with neighbour galaxies seem to be a driving force in the evolution of the neutral gas component in many early-type disk galaxies.

- Many early-type disk galaxies have lopsided morphologies. In most cases the asymmetries can be explained as the result of interaction, accretion or merger events. Few isolated galaxies have lopsided gas distributions.

- Kinematic lopsidedness is rare in early-type disk galaxies, even in interacting systems.

The data presented in the atlas in the appendix form the basis for a study of the rotation curves and dark matter content in these early-type disk galaxies. This study is currently ongoing, and the results will be presented in a forthcoming paper.

Acknowledgements. Great appreciation goes to the members of the WHISP team, in particular Yuan Tang, for carrying out the crucial first stages of the data reduction of the WHISP observations. EN is grateful to the Department of Physics and
Astronomy of the University of Sheffield for the hospitality during part of this work. The Westerbork Synthesis Radio Telescope is operated by ASTRON (Netherlands Foundation for Research in Astronomy) with support from The Netherlands Foundation for Scientific Research (NWO). We made extensive use of data from the Lyon Extragalactic Database (LEDA; http: //leda. univ-lyon1. fr/) and the NASA/IPAC Extragalactic Database (NED; http://nedwww.ipac.caltech.edu/) which is operated by the Jet Propulsion Laboratory, California Institute of Technology, under contract with the National Aeronautics and Space Administration. This research was funded by NWO grant no. 614031009 .

\section{References}

Allsopp, N. J. 1979, MNRAS, 187, 537

Baldwin, J. E., Lynden-Bell, D., \& Sancisi, R. 1980, MNRAS, 193, 313

Battinelli, P., Capuzzo-Dolcetta, R., Hodge, P. W., Vicari, A., \& Wyder, T. K. 2000, A\&A, 357, 437

Bosma, A. 1978, Ph.D. Thesis, Rijksuniversiteit Groningen

Bosma, A. 1981, AJ, 86, 1791

Bosma, A., Ekers, R. D., \& Lequeux, J. 1977, A\&A, 57, 97

Braun, R. 1997, ApJ, 484, 637

Braun, R., Walterbos, R. A. M., \& Kennicutt, R. C. 1992, Nature, 360, 442

Broeils, A. H. 1992, Ph.D. Thesis, Rijksuniversiteit Groningen

Broeils, A. H., \& Rhee, M.-H. 1997, A\&A, 324, 877

Broeils, A. H., \& van Woerden, H. 1994, A\&AS, 107, 129

Buta, R., van Driel, W., Braine, J., et al. 1995, ApJ, 450, 593

Carignan, C., \& Freeman, K. C. 1988, ApJ, 332, L33

Carilli, C. L., \& van Gorkom, J. H. 1992, ApJ, 399, 373

Carilli, C. L., van Gorkom, J. H., \& Stocke, J. T. 1989, Nature, 338, 134

Cayatte, V., Kotanyi, C., Balkowski, C., \& van Gorkom, J. H. 1994, AJ, 107, 1003

Cox, A. N. 2000, Allen's astrophysical quantities, 4th ed. (New York: Springer)

Cuillandre, J., Lequeux, J., Allen, R. J., Mellier, Y., \& Bertin, E. 2001, ApJ, 554, 190

de Blok, W. J. G., McGaugh, S. S., \& van der Hulst, J. M. 1996, MNRAS, 283, 18

Faber, S. M., \& Gallagher, J. S. 1979, ARA\&A, 17, 135

Falco, E. E., Kurtz, M. J., Geller, M. J., et al. 1999, PASP, 111, 438

Ferguson, A. M. N., Wyse, R. F. G., Gallagher, J. S., \& Hunter, D. A. 1998, ApJ, 506, L19

Garcia, A. M. 1993, A\&AS, 100, 47

García-Ruiz, I., Sancisi, R., \& Kuijken, K. 2002, A\&A, 394, 769

Hameed, S., \& Young, L. M. 2003, AJ, 125, 3005

Haynes, M. P., Jore, K. P., Barrett, E. A., Broeils, A. H., \& Murray, B. M. 2000, AJ, 120, 703

Haynes, M. P., van Zee, L., Hogg, D. E., Roberts, M. S., \& Maddalena, R. J. 1998, AJ, 115, 62

Heckman, T. M., Sancisi, R., Balick, B., \& Sullivan, W. T. 1982, MNRAS, 199, 425

Hickson, P., Kindl, E., \& Auman, J. R. 1989, ApJS, 70, 687

Jog, C. J. 1997, ApJ, 488, 642

Jore, K. P. 1997, Ph.D. Thesis, Cornell University

Kamphuis, J. J., Sijbring, D., \& van Albada, T. S. 1996, A\&AS, 116, 15

Keel, W. C., \& Hummel, E. 1988, A\&A, 194, 90

Kennicutt, R. C. 1989, ApJ, 344, 685 
Knapp, G. R., van Driel, W., Schwarz, U. J., van Woerden, H., \& Gallagher, J. S. 1984, A\&A, 133, 127

Koski, A. T. 1978, ApJ, 223, 56

Lucy, L. B. 1974, AJ, 79, 745

Martin, C. L., \& Kennicutt, R. C. 2001, ApJ, 555, 301

Matthews, L. D., van Driel, W., \& Gallagher, J. S. 1998, AJ, 116, 1169

Mundell, C. G., Pedlar, A., Shone, D. L., \& Robinson, A. 1999, MNRAS, 304, 481

Nilson, P. 1973, Uppsala general catalogue of galaxies, Uppsala Astr. Obs. Ann., Vol. 6 (UGC)

Noordermeer, E., Sparke, L. S., \& Levine, S. E. 2001, MNRAS, 328, 1064

Oosterloo, T., \& Shostak, S. 1993, A\&AS, 99, 379

Pedlar, A., Howley, P., Axon, D. J., \& Unger, S. W. 1992, MNRAS, 259, 369

Persic, M., Salucci, P., \& Stel, F. 1996, MNRAS, 281, 27

Pogge, R. W. 1989, ApJS, 71, 433

Pogge, R. W., \& Eskridge, P. B. 1993, AJ, 106, 1405

Regan, M. W., Thornley, M. D., Helfer, T. T., et al. 2001, ApJ, 561, 218

Richter, O.-G., \& Sancisi, R. 1994, A\&A, 290, L9

Rix, H. \& Zaritsky, D. 1995, ApJ, 447, 82

Roberts, M. S., \& Haynes, M. P. 1994, ARA\&A, 32, 115

Rosenberg, J. L., \& Schneider, S. E. 2000, ApJS, 130, 177

Rubin, V. C. 1994, AJ, 107, 173

Sancisi, R., van Woerden, H., Davies, R. D., \& Hart, L. 1984, MNRAS, 210, 497

Schaye, J. 2004, ApJ, 609, 667

Schiminovich, D., van Gorkom, J. H., van der Hulst, J. M., \& Kasow, S. 1994, ApJ, 423, L101

Schiminovich, D., van Gorkom, J. H., van der Hulst, J. M., \& Malin, D. F. 1995, ApJ, 444, L77

Schwarz, U. J. 1978, A\&A, 65, 345

Seyfert, C. K. 1943, ApJ, 97, 28

Shane, W. W. 1980, A\&A, 82, 314

Simkin, S. M., Su, H., van Gorkom, J., \& Hibbard, J. 1987, Science, 235, 1367

Smith, B. J. 1994, AJ, 107, 1695

Sofue, Y., Honma, M., \& Arimoto, N. 1995, A\&A, 296, 33

Sofue, Y., Koda, J., Nakanishi, H., et al. 2003, PASJ, 55, 17

Swaters, R. 1999, Ph. D. Thesis, Rijksuniversiteit Groningen

Swaters, R. A., \& Balcells, M. 2002, A\&A, 390, 863 (Paper II)

Swaters, R. A., Schoenmakers, R. H. M., Sancisi, R., \& van Albada, T. S. 1999, MNRAS, 304, 330
Swaters, R. A., van Albada, T. S., van der Hulst, J. M., \& Sancisi, R. 2002, A\&A, 390, 829 (Paper I)

Thilker, D. A., Bianchi, L., Boissier, S., et al. 2005, ApJ, 619, L79

Toomre, A. 1964, ApJ, 139, 1217

Tully, R. B. 1980, ApJ, 237, 390

Tully, R. B. 1988, Nearby galaxies catalog (Cambridge and New York: Cambridge University Press), 221

van Albada, T. S. \& Sancisi, R. 1986, Royal Society of London Philosophical Transactions Series A, 320, 447

van der Hulst, J. M., van Albada, T. S., \& Sancisi, R. 2001, in Gas and Galaxy Evolution, ASP Conf. Ser. 240, 451

van der Marel, R. P., \& Franx, M. 1993, ApJ, 407, 525

van Driel, W. 1987, Ph. D. Thesis, Rijksuniversiteit Groningen

van Driel, W., \& Buta, R. J. 1991, A\&A, 245, 7

van Driel, W., \& van Woerden, H. 1991, A\&A, 243, 71

van Driel, W., \& van Woerden, H. 1994, A\&A, 286, 395

van Driel, W., van Woerden, H., Schwarz, U. J., \& Gallagher, J. S. 1988, A\&A, 191, 201

van Driel, W., Balkowski, C., \& van Woerden, H. 1989, A\&A, 218, 49

van Moorsel, G. A. 1983a, A\&AS, 53, 271

van Moorsel, G. A. 1983b, A\&AS, 54, 1

van Moorsel, G. A. 1988, A\&A, 202, 59

van Woerden, H., van Driel, W., \& Schwarz, U. J. 1983, in Internal Kinematics and Dynamics of Galaxies, IAU Symp. 100, 99

van Zee, L., Haynes, M. P., Salzer, J. J., \& Broeils, A. H. 1997, AJ, 113,1618

Verdes-Montenegro, L., Bosma, A., \& Athanassoula, E. 1995, A\&A, 300,65

Verheijen, M. A. W. 2001, ApJ, 563, 694

Verheijen, M. A. W., \& Sancisi, R. 2001, A\&A, 370, 765

Vogelaar, M. G. R., \& Terlouw, J. P. 2001, in Astronomical Data Analysis Software and Systems X, ASP Conf. Ser. 238, 358

Vorontsov-Vel'Yaminov, B. A. 1977, A\&AS, 28, 1

Warmels, R. H. 1988a, A\&AS, 73, 453

Warmels, R. H. 1988b, A\&AS, 72, 427

Wevers, B. M. H. R., Appleton, P. N., Davies, R. D., \& Hart, L. 1984, A\&A, 140, 125

Wilcots, E. M., \& Prescott, M. K. M. 2004, AJ, 127, 1900

Williams, B. A., McMahon, P. M., \& van Gorkom, J. H. 1991, AJ, 101, 1957

Wong, T., \& Blitz, L. 2002, ApJ, 569, 157 